\title{
Identification of Mubritinib (TAK 165) as an inhibitor of KSHV driven primary effusion lymphoma via disruption of mitochondrial OXPHOS metabolism
}

\author{
Abram Calderon ${ }^{1}$, Samantha S. Soldan ${ }^{1}$, Alessandra De Leo ${ }^{1}$, Zhong Deng ${ }^{1}$, \\ Drew M. Frase ${ }^{1}$, Emma M. Anderson ${ }^{1}$, Yue Zhang ${ }^{1}$, Olga Vladimirova ${ }^{1}$, Fang Lu ${ }^{1}$, \\ Jessica C. Leung ${ }^{1}$, Maureen E. Murphy ${ }^{1}$ and Paul M. Lieberman ${ }^{1}$ \\ ${ }^{1}$ The Wistar Institute, Philadelphia, PA 19146, USA \\ Correspondence to: Paul M. Lieberman, email: lieberman@wistar.org \\ Keywords: KSHV; LANA; high-throughput screen (HTS); primary effusion lymphoma; Mubritinib \\ Received: September 24, 2020 Accepted: October 29, $2020 \quad$ Published: November 17, 2020 \\ Copyright: ( 2020 Calderon et al. This is an open access article distributed under the terms of the Creative Commons Attribution License \\ (CC BY 3.0), which permits unrestricted use, distribution, and reproduction in any medium, provided the original author and source are credited.
}

\section{ABSTRACT}

KSHV-associated cancers have poor prognoses and lack therapeutics that selectively target viral gene functions. We developed a screening campaign to identify known drugs that could be repurposed for the treatment of KSHV-associated cancers. We focused on primary effusion lymphoma (PEL), which has particularly poor treatment outcomes. We developed a luciferase reporter assay to test the ability of drugs to inhibit DNA binding of the KSHV LANA DNA binding domain (DBD). In parallel, we screened drugs for selective inhibition of a KSHV+ PEL cells. While potent hits were identified in each assay, only one hit, Mubritinib, was found to score in both assays. Mubritinib caused PEL cells to undergo cell cycle arrest with accumulation of sub-G ${ }_{1}$ population and Annexin V. Mubritinib inhibited LANA binding to KSHV terminal repeat (TR) DNA in KSHV+ PEL cells, but did not lead to KSHV lytic cycle reactivation. Mubritinib was originally identified as a receptor tyrosine kinase (RTK) inhibitor selective for HER2/ErbB2. But recent studies have revealed that Mubritinib can also inhibit the electron transport chain (ETC) complex at nanomolar concentrations. We found that other related ETC complex inhibitors (Rotenone and Deguelin) exhibited PEL cell growth inhibition while RTK inhibitors failed. Seahorse analysis demonstrated that Mubritinib selectively inhibits the maximal oxygen consumption (OCR) in PEL cells and metabolomics revealed changes in ATP/ADP and ATP/AMP ratios. These findings indicate that PEL cells are selectively sensitive to ETC complex inhibitors and provide a rationale for repurposing Mubritinib for selective treatment of PEL.

\section{INTRODUCTION}

Kaposi's Sarcoma-associated Herpesvirus (KSHV)/ Human Herpesvirus 8 (HHV8) is a human $\gamma$-herpesvirus that establishes latent infection in B-lymphocytes and is strongly associated with several human cancers [1-3]. KSHV DNA and latency gene products are consistently detected in all forms of Kaposi's sarcoma (KS), most forms of primary effusion lymphoma (PEL) and multicentric Castleman's disease (MCD), and a percentage of B-cell lymphoproliferative diseases [4-6]. Introduction of KSHV DNA into mice causes KS-like malignancies, demonstrating that KSHV genes associated with latent infection promote the development of KS-like tumors
[7-10]. KSHV is therefore considered the causative agent of KS and essential for the formation of PEL and MCD. At present, there are no KSHV-specific therapeutics approved for treatment of KSHV-associated cancers.

KSHV cancers consistently express a limited number of viral proteins that are associated with latent infection. During latency the KSHV genome is maintained by the viral Latency-Associated Nuclear Antigen (LANA), encoded by ORF73 [11-13]. LANA is a multifunction protein that plays a role in viral and cellular gene regulation, DNA replication, chromosome organization, cell cycle progression, and cell survival [14-16]. LANA, like EBNA1 from the Epstein Barr virus (EBV), is a DNA binding protein that binds and maintains viral genomes 
during latent infection [17]. LANA can also repress viral lytic genes, like ORF50 (RTA), and autoregulate its own transcription to maintain a stable latent infection $[17,18]$. LANA has been shown to activate transcription of cellular oncogenes, such as hTERT, and repress tumor suppressors, like $\mathrm{Rb}$, to promote oncogenic transformation $[19,20]$. Furthermore, LANA is consistently detected in all KSHVassociated tumors [21]. Therefore, LANA is an attractive target for the development of KSHV-specific cancer therapeutics.

The mechanisms through which LANA maintains $\mathrm{KSHV}$ episomes during latent infection is known in some detail $[14,16]$. LANA binds directly to a 20 bp GCrich sequence within the KSHV terminal repeats (TRs) [11-13, 22-24]. A minimal region of $\sim 60$ bp containing two LANA binding sites is sufficient to support transient DNA replication of plasmids [22]. Long-term episome maintenance requires an additional sequence from the TR and the presence of at least two TRs [24]. The C-terminal domain of LANA possesses sequence-specific DNA binding activity to the 18 bp element in the KSHV minimal replicator [22]. The X-ray structure of the DNA binding domain has been solved alone $[25,26]$ and in complex with bound TR DNA [27]. The LANA amino terminal domain interacts with histone $\mathrm{H} 2 \mathrm{~A} / \mathrm{H} 2 \mathrm{~B}$ to tether itself to the metaphase chromosomes, which is required for episome maintenance [28]. Small molecules that bind to LANA have been explored [29] and identified [30-32]. But, to date, none of these have yet demonstrated efficacy in vivo.

In an effort to find more efficacious treatments for KSHV-associated diseases, we have initiated a cell-based small-molecule screening campaign to identify inhibitors of LANA DNA binding that also inhibit KSHV positive cell growth. While LANA remains an attractive target for inhibition of KSHV-associated cancers, loss of LANA may not be sufficient to eradicate KSHV cancer cell growth. Genetic disruption of KSHV LANA leads to loss of viral episomes $[33,34]$, but it is not yet clear if this loss leads to an inhibition of cancer cell growth or tumorigenesis. Therefore, we set out to identify small molecule inhibitors of LANA DNA-binding that could also be readily tested for their ability to inhibit KSHV positive cell growth and tumorigenicity (Figure 1). To accomplish this, we screened a library of known drugs that could be repurposed for the treatment of patients with KSHV-associated cancers and related diseases.

\section{RESULTS}

\section{Development of a cell-based assay for inhibition of LANA DNA binding}

We first developed a cell-based luciferase assay that would allow us to screen small molecules for their ability to inhibit LANA DNA binding (Figure 2A). To this end, we used the RMCE-HILO platform (41) to generate a stable HEK293T cell line that expresses a FLAG-tagged fusion protein containing the activation domain of the herpes simplex virus transcription factor VP16 and the LANA DNA-binding domain (DBD) upon induction with doxycycline (Figure 2B). We also generated a Gaussia luciferase reporter plasmid containing the three known LANA binding sites (LBS2, LBS1, and LBS3) from the KSHV TR region. In the presence of the LBS reporter plasmid, the fusion protein can bind to the LANA binding sites and transactivate expression of Gaussia luciferase. The extent of activation was sufficiently robust ( $\sim 14$-fold) for high-throughput screening. However, if an appropriate inhibitor is added to the media, the binding of the fusion protein will be reduced, causing a reduction in Gaussia luciferase expression.

\section{Identification of drugs that inhibit LANA DNA binding}

The luciferase assay was used to screen a small library $(\sim 1,000$ known drugs $)$ from SelleckChem at a final concentration of $12.5 \mu \mathrm{M}$. Although a large number of these drugs had little effect on either cell viability or luciferase signal (Figure 2C, upper right cluster), there were also many drugs for which efficacy was more difficult to interpret. Therefore, we selected hits based on the inhibition of the luciferase signal relative to cell viability. This "therapeutic window" was calculated as the percent cell viability relative to DMSO control minus the percent luciferase signal relative to DMSO control (Figure $2 \mathrm{D}$ and $2 \mathrm{E}$ ). Among the top hits, were several receptor tyrosine kinase (RTK) inhibitors, including Linifanib, Regorafenib, MGCD-265, BMS777606, and Mubritinib, and the ROCK-inhibitor GSK429286A (Figure 2E). We concluded that these drugs inhibit LANA DNA binding at micromolar concentrations in living cells.

\section{Screening for drugs that inhibit $\mathrm{KSHV}^{+}$cell growth}

In parallel, we also screened the same SelleckChem library at a final concentration of $10 \mu \mathrm{M}$ against two B-cell lines, a KSHV cell line (Ramos) and a $\mathrm{KSHV}^{+} \mathrm{PEL}$ cell line (BC3) (Figure 3). As expected, most of the drugs showed similar effects on the viability of both of these cell lines (Figure 3A). Nevertheless, there were a small number of drugs that selectively reduced the viability of the $\mathrm{KSHV}^{+} \mathrm{BC} 3$ cells relative to Ramos cells (Figure 3B). As with the luciferase assay, we chose hits based on a "therapeutic window", which is simply the Ramos $\left(\mathrm{KSHV}^{-}\right)$percent cell viability minus the $\mathrm{BC} 3\left(\mathrm{KSHV}^{+}\right)$ percent cell viability, and the reproducibility of the $\mathrm{BC} 3$ viability signal (from data acquired on two separate days) (Figure 3C). Using this approach, we identified several drugs that selectively inhibited $\mathrm{BC} 3$ cell growth relative 
to Ramos cells (Figure 3C and 3D). Mubritinib was the only drug that appeared as a hit in both of the screens, even though its therapeutic window was relatively small.

\section{Mubritinib selectively inhibits PEL cell viability at nanomolar concentrations}

Hits from both the luciferase and B-cell viability screens were assayed against a panel of six B-cell lines (Figure 3E) at ten different concentrations (Figure 3F). These data were used to determine growth inhibition index $\left(\mathrm{GI}_{50}\right)$ values for each of these drugs (Figure $3 \mathrm{G}$, Supplementary Table 1). Interestingly, only Mubritinib was found to reproducibly and selectively reduce the viability of $\mathrm{KSHV}^{+} \mathrm{PEL}$ cell lines (BC3, BCBL1, and $\mathrm{BC} 1)$ relative to $\mathrm{KSHV}^{-}$cell lines $(\mathrm{BJAB}$, Ramos, and LCL352) at nanomolar concentrations (Figure 3G). In particular, the $\mathrm{GI}_{50}$ values for Mubritinib in BC3, BCBL1, and $\mathrm{BC} 1$ cells were $13.45,17.1$, and $7.5 \mathrm{nM}$, respectively, while the $\mathrm{GI}_{50}$ values for BJAB, Ramos, and LCL352 cells were of $1.6,0.4$, and $0.2 \mu \mathrm{M}$, respectively. The results for the LCL352 cells are notable since these cells are infected with EBV, a $\gamma$-herpesvirus that is closely related to KSHV.

\section{Comparison of Mubritinib with other published PEL inhibitors}

After identifying Mubritinib as a hit, we sought to compare the selectivity that we observed with this drug to other published drugs that have been tested against PEL cell lines. In particular, we chose to compare Mubritinib to cytarabine (CYT) [35] and rapamycin (RAP) [36]. We began by testing the effects of these drugs on the cell cycle using propidium iodide staining and flow cytometry (Figure 4A and 4B, Supplementary Figure 1). For these experiments, we chose to test each drug at concentrations similar to those that have been previously published $[35,36]$. DMSO and camptothecin (CPT) were used as negative and positive controls, respectively. When BJAB and LCL352 cells were treated with $7.5 \mathrm{nM}$ Mubritinib, the total population of $\mathrm{S}$ and $\mathrm{G} 2$ cells was similar to that observed when these cells were treated with DMSO (Figure 4D). However, when PEL cells (BC1 and BCBL1) were treated with the same concentration of Mubritinib, a significant decrease in the total population of $\mathrm{S}$ and G2 cells (Figure 4A), and corresponding increase in G1+subG1 (Supplementary Figure 1) was observed.
Screen A

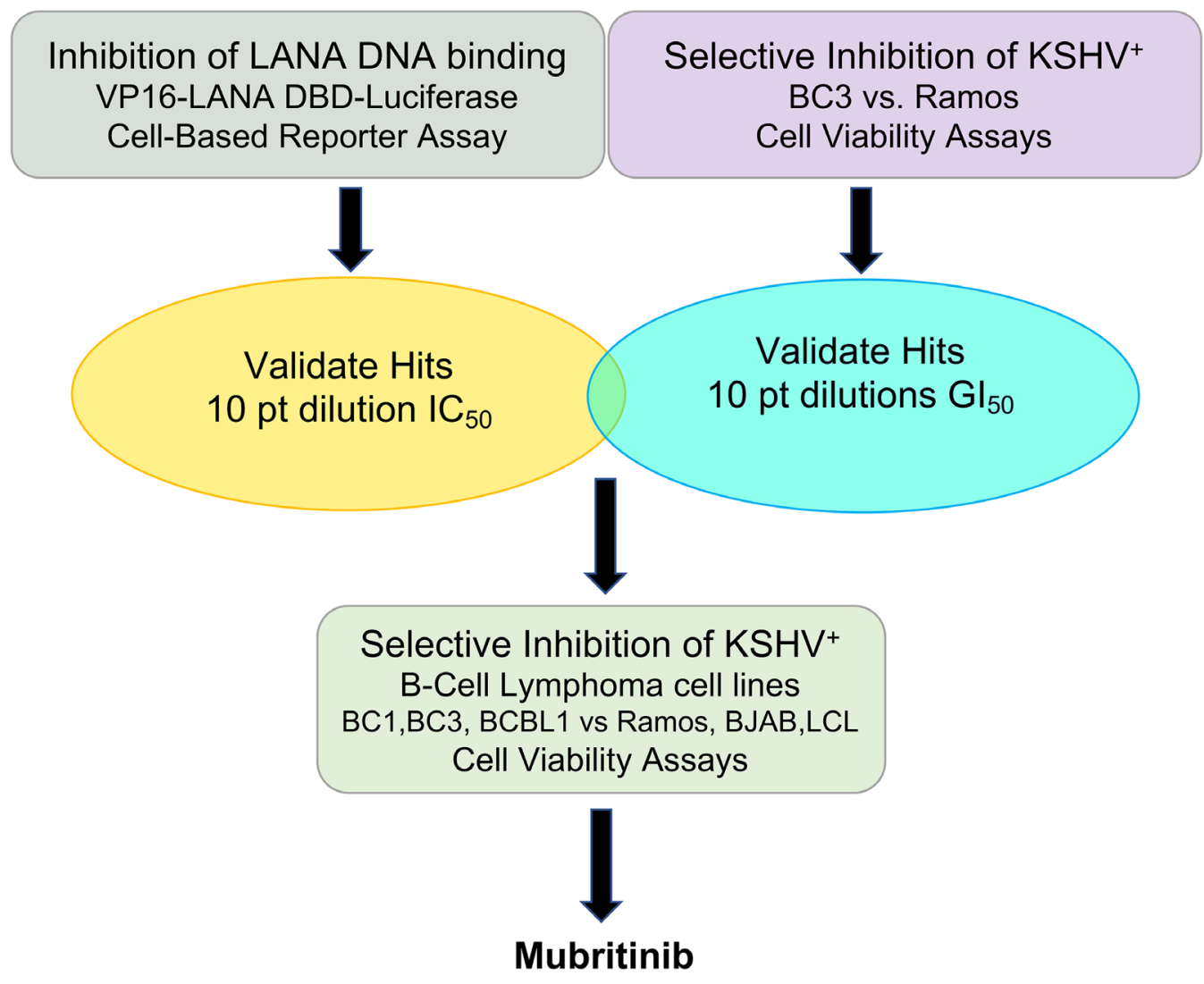

Figure 1: Drug screening strategy. Two primary screens one testing for inhibition of LANA DNA binding (Screen A) and another testing for selective cell growth inhibition of $\mathrm{KSHV}^{+} \mathrm{B}$-cell lymphoma (Screen B), were performed in parallel to identify LANA-specific inhibitors of KSHV positive tumors. Then, hits from these two screens were titrated against a panel of 6 B-cell lines. Mubritinib was identified as the best drug for further testing in $\mathrm{KSHV}^{+}$PEL cells. 
In contrast, neither cytarabine nor rapamycin showed selective inhibition of $\mathrm{KSHV}^{+}$PEL cell growth as did Mubritinib (Figure 4A and Supplementary Figure 1).

\section{Mechanism of action of Mubritinib in PEL cells}

To begin to understand the mechanism of action of Mubritinib, we first analyzed the cell cycle profiles for two $\mathrm{KSHV}^{+}$PELs (BC1 and BCBL1) and two $\mathrm{KSHV}^{-}$ lymphomas (BJAB and LCL352) after treatment with $15 \mathrm{nM}$ Mubritinib (Figure 4B). Mubritinib treatment altered the cell cycle profiles of $\mathrm{BC} 1$ and BCBL1 cells, while having no detectable effects on the cell cycle profiles of BJAB or LCL352 cells. For the two PEL cell lines (BC1 and BCBL1), Mubritinib reduced S and G2 populations and increased the sub- $\mathrm{G}_{1}$ population (Figure 4B). To further investigate these effects on cell viability markers, we next performed flow cytometry with Annexin V/PI binding analysis (Figure 4C and 4D). Treatment with $15 \mathrm{nM}$ Mubritinib caused higher decreases in live and increases in apoptotic cell populations for the PEL cell lines (BC1 and BCBL1) compared to $\mathrm{BJ} A B$ and LCL352 cells (Figure 4D). We also compared the effects of Mubritinib with cytarabine and camptothecin (Figure 4D). Only Mubritinib, and not cytarabine or camptothecin, showed strong selectivity for PEL cells when scored for percentage of live cells.

To assess whether Mubritinib can inhibit the binding of LANA to KSHV genomes, we performed chromatinimmunoprecipitation (ChIP) assays in BCBL1 cells (Figure 4E). We found that LANA binding to the TR was reduced $\sim 50 \%$ in BCBL1 cells treated with $15 \mathrm{nM}$ Mubritinib for $72 \mathrm{hrs}$. LANA did not bind to negative control regions of the viral or cellular genome, indicating specificity in the LANA ChIP assay. Furthermore, Mubritinib did not induce lytic activator RTA at either 48 or $90 \mathrm{hrs}$ of treatment, as measured by Western blot analysis (Figure 4F) or RT-qPCR of KSHV lytic cycle genes (Supplementary Figure 2). Interestingly there was a modest down regulation of LANA by Western blot (Figure
A

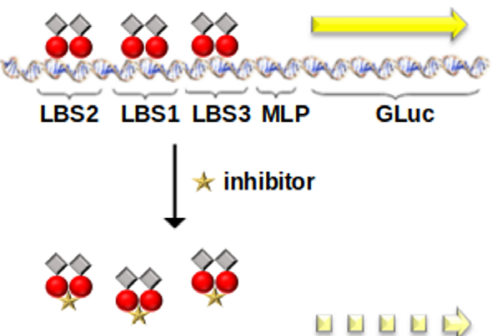

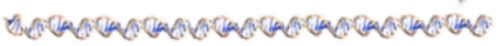

B

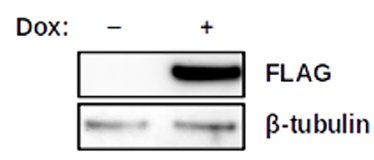

C

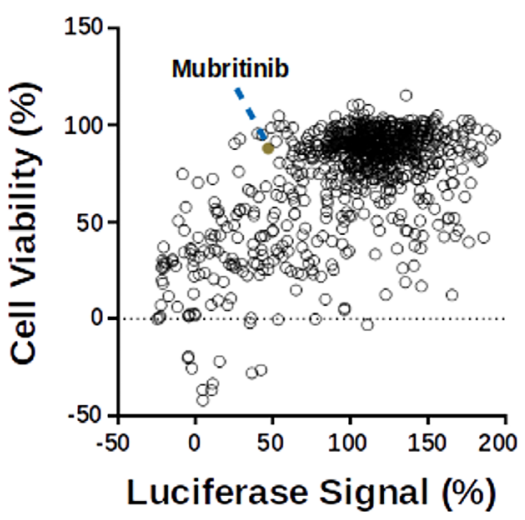

D

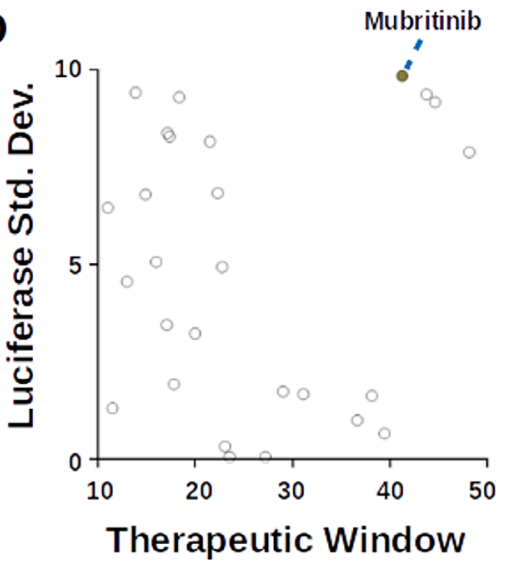

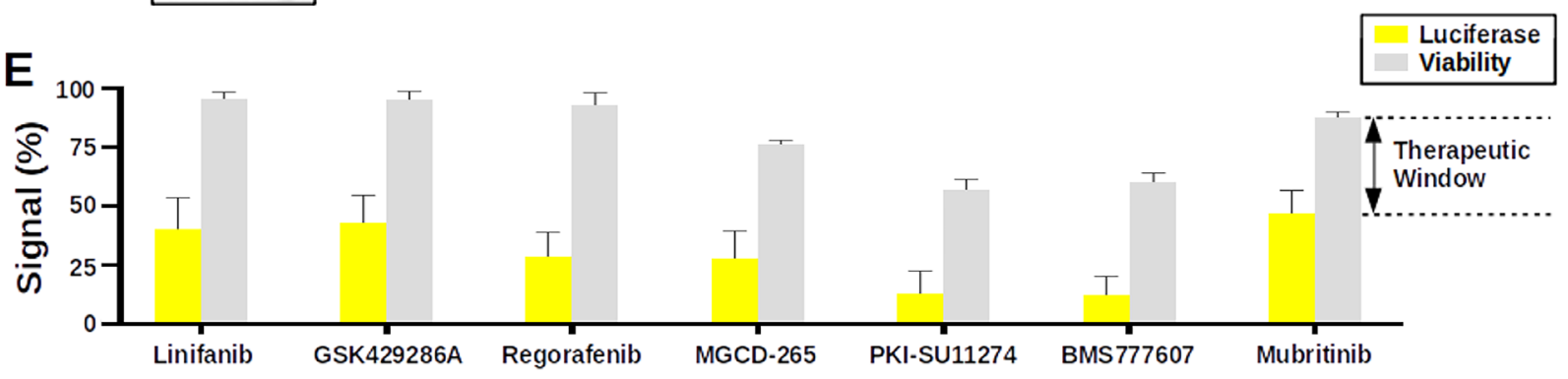

Figure 2: Cell-based screen for inhibitors of LANA DNA-binding. (A) Cartoon illustrating key features of the Gaussia luciferase assay. The reporter plasmid has three LANA Binding Sites (LBSs) adjacent to a Major Late Promoter (MLP) for the Gaussia luciferase (GLuc) gene. A fusion protein containing the Vp16 activation domain (gray diamond) and the LANA DNA-binding domain (DBD) (red circle) can bind to the LANA binding sites and enhance expression of GLuc (yellow arrow). However, in the presence of an inhibitor (gold star), LANA DNA-binding is reduced, causing a reduction in GLuc expression (dashed arrow). (B) Western blot demonstrating that expression of the FLAG-tagged Vp16-LANA DBD fusion protein is induced by doxycycline. Tubulin was used as a loading control. (C) Scatter plot showing the results of the luciferase screen. A large number of drugs (open circles) cluster near the upper right region of the graph, indicating that they had little effect on either the luciferase signal or the cell viability. Mubritinib is highlighted as a filled red circle. (D) Scatter plot focusing on drugs from the luciferase screen that have high therapeutic windows and highly reproducible luciferase signals. Mubritinib is highlighted as a filled red circle. (E) Graph summarizing data for top hit compounds from the luciferase screen as percentage of signal with DMSO control for Luciferase (yellow) or Resazurin viability assay (grey) relative to DMSO controls. The therapeutic index is calculated as the difference between the Resazurin and Luciferase signals. 
4F) and ORF73 mRNA expression as measured by RTPCR (Supplementary Figure 2) after 48 hours treatment of BCBL1 cells. Taken together, these findings indicate that nanomolar concentrations of Mubritinib cause cell cycle arrest with accumulation of sub- $\mathrm{G}_{1}$ cell populations selective for $\mathrm{KSHV}^{+} \mathrm{PEL}$ cells, and partially disrupt LANA binding to TR.

\section{Mubritinib also exhibits selectivity in vivo}

Given the potency and selectivity that Mubritinib displayed for inhibition of PEL cell growth in culture, we decided to examine the effects of this drug in two PEL mouse models (Figure 5A). For comparison, we chose to implant mice with $\mathrm{EBV}^{+}$LCL352 B-cells. All of the xenografts were transduced with mCherry/eLuciferase for in vivo imaging. $\mathrm{BCBL} 1$ and $\mathrm{BC} 1$ mice treated with vehicle rapidly gained weight due to xenograft growth (Figure 5B). However, this increase was significantly slowed in mice treated once daily with $25 \mathrm{mg} / \mathrm{kg}$ Mubritinib. By comparison, LCL352 mice treated with Mubritinib exhibited weights similar to those treated with vehicle. All of the mice that were treated with vehicle developed strong bioluminescence signals (Figure 5C and 5D). However,
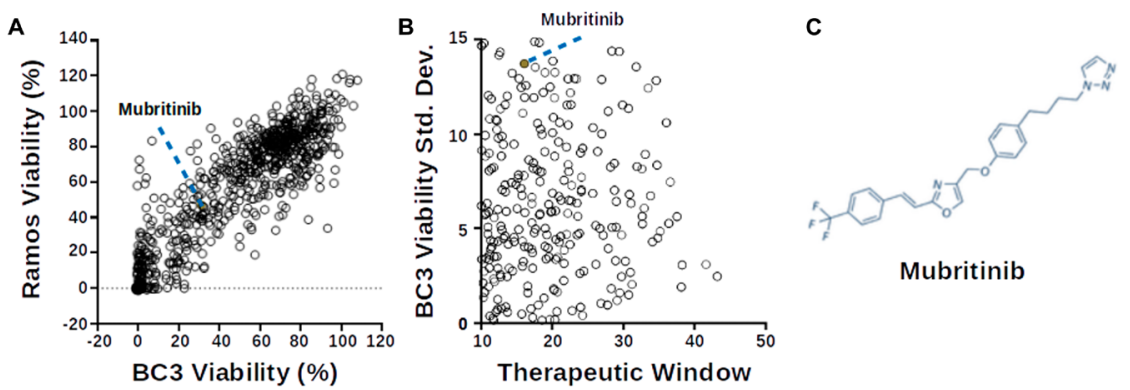

D
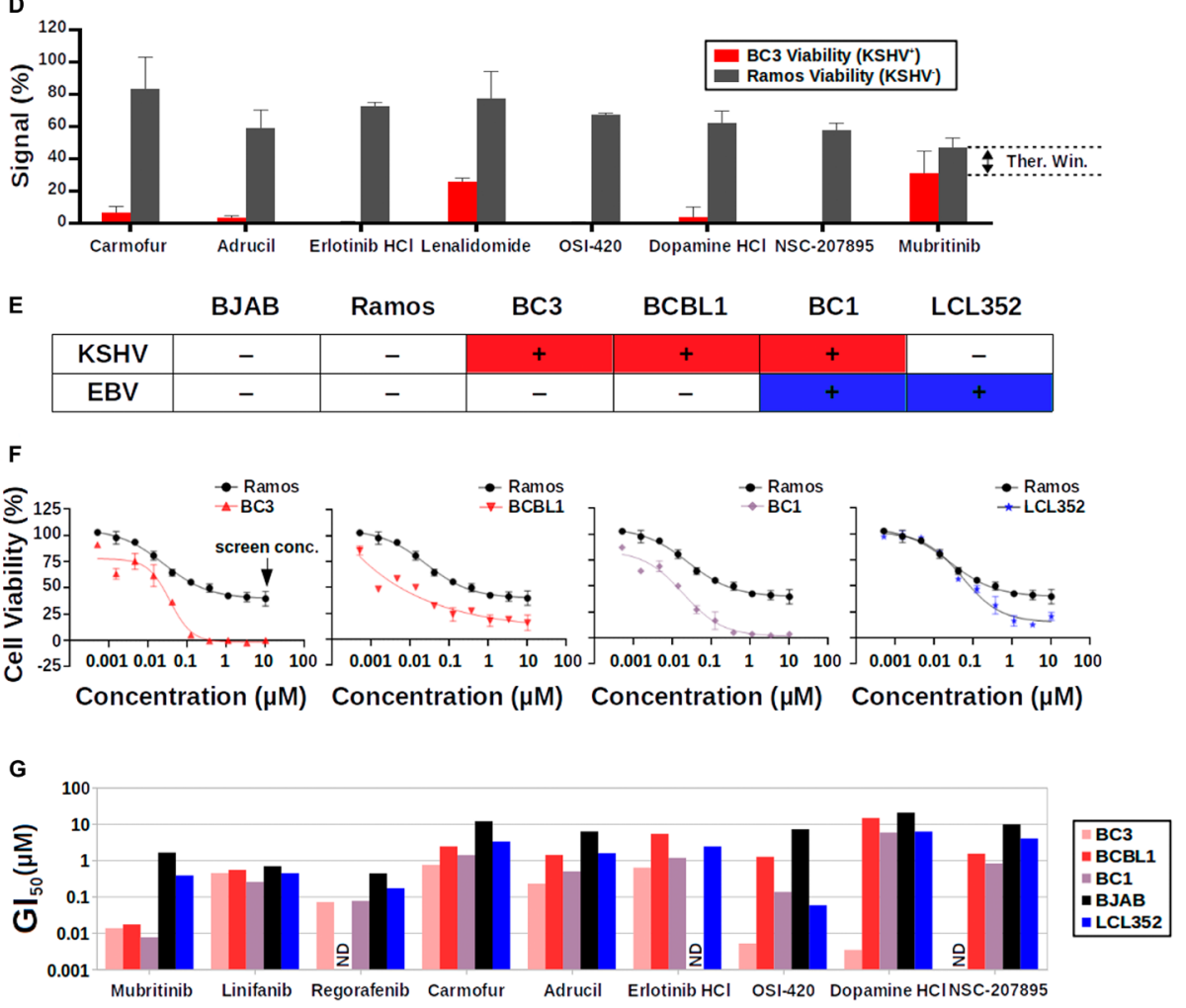

Figure 3: Cell-based screen for selective inhibition of PEL cell growth. (A) Scatter plot of the results of the cell growth inhibition screen. A large number of drugs (open circles) cluster along a diagonal line, indicating that they show similar effects on the viability of both Ramos $\left(\mathrm{KSHV}^{-}\right)$and $\mathrm{BC} 3\left(\mathrm{KSHV}^{+}\right)$cells. Mubritinib is highlighted as a filled red circle. (B) Scatter plot focusing on drugs from the cell growth inhibition screens that have high therapeutic windows and highly reproducible BC3 viability signals. Mubritinib is highlighted as a filled red circle. (C) Chemical structure of Mubritinib. (D) Graph summarizing data for hit compounds from the cell growth inhibition screen. (E) Table summarizing the KSHV and EBV infection status of the B-cell lines used in this paper. (F) Titration of Mubritinib against various B-cell lines. Ramos (KSHV-, $\left.\mathrm{EBV}^{-}\right)$data are shown for reference in each graph. $\mathrm{KSHV}^{+}$cell lines $(\mathrm{BC}$, BCBL1, and BC1) respond more strongly to Mubritinib at nanomolar concentrations than the $\mathrm{EBV}^{+}\left(\mathrm{LCL}_{52}\right)$ cell line. (G) Graph summarizing the $\mathrm{GI}_{50}$ values for hits from the SelleckChem Library. 
A

BJAB

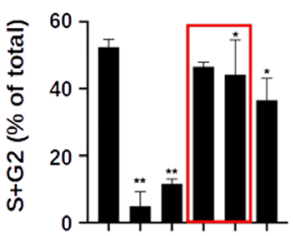

DMSO $\quad+$\begin{tabular}{ll|l|l|l|l|}
\hline & - & - & - & - & - \\
\hline
\end{tabular}

\begin{tabular}{l|l|l|l|l|l|l|}
$\operatorname{CPT}(4 \mu \mathrm{MM})$ & + & - & - & - & - & \\
\cline { 2 - 7 } & - & + & - & - & - & - \\
\hline
\end{tabular}

CYT (1 $\mu \mathrm{M})$

$\operatorname{MUB}(7.5 \mathrm{nM})$

MUB (15 nM)

RAP ( $40 \mathrm{nM}$ )

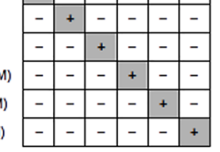

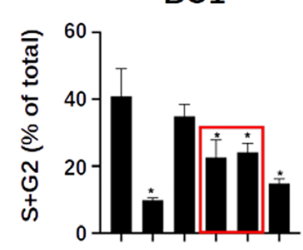
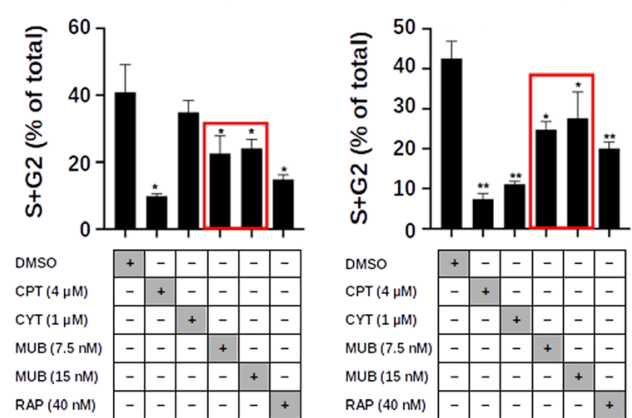

B
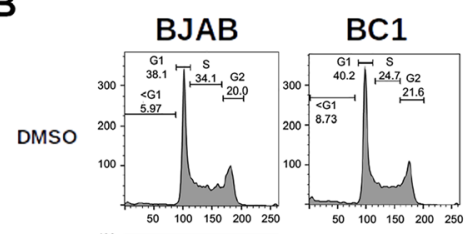

BCBL1
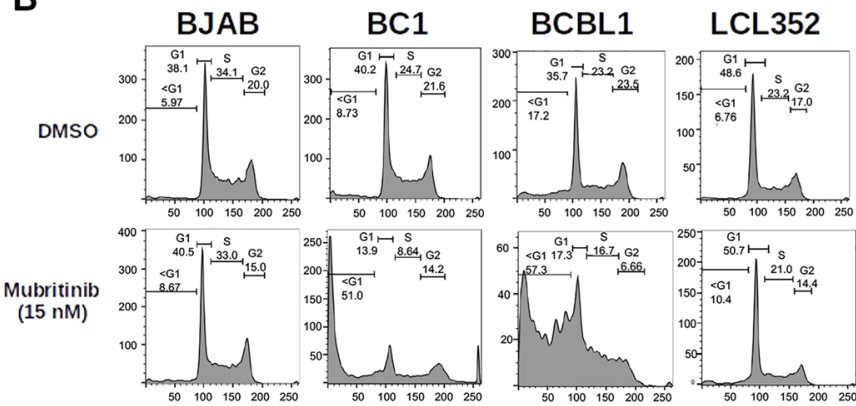

C
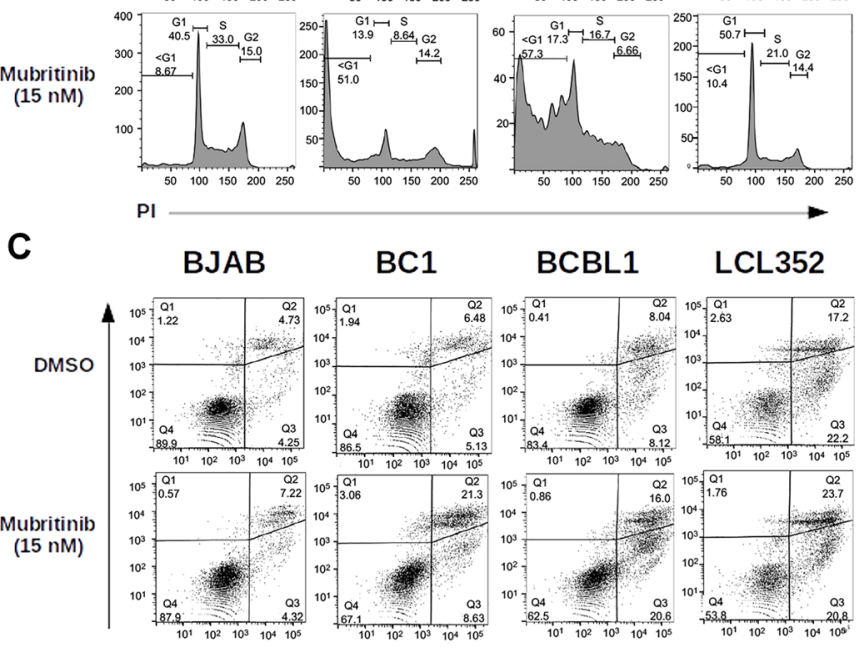

a ANNEXIN V

E

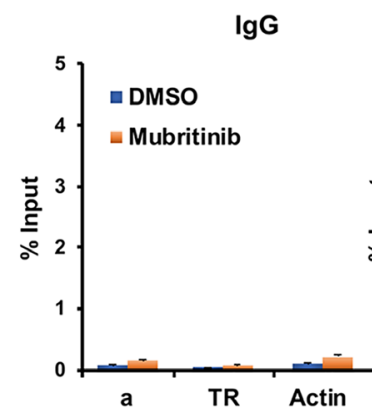

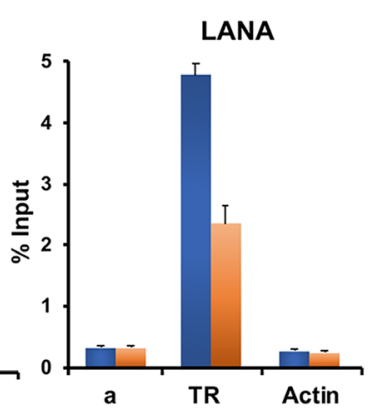

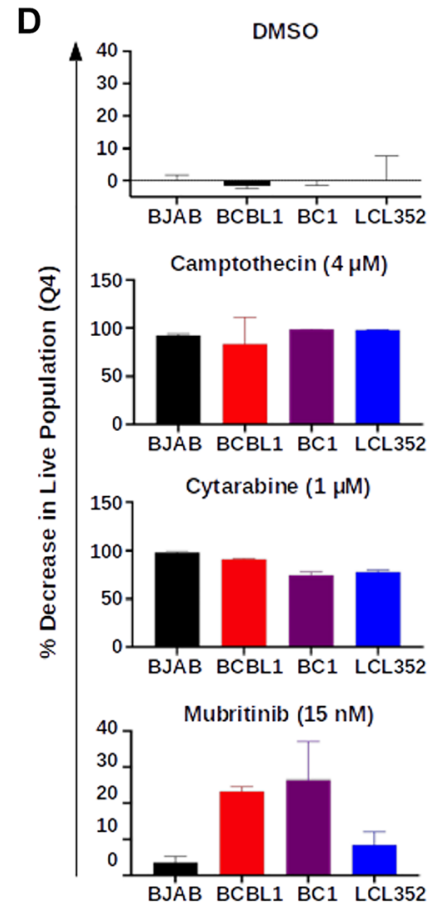

$\mathbf{F}$

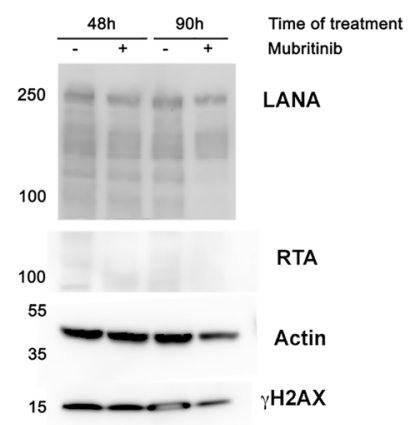

Figure 4: Mubritinib inhibits PEL cell cycle progression and LANA DNA binding. (A) Graph of cell cycle kinetics data comparing Mubritinib (MUB) to cytarabine (CYT) and rapamycin (RAP). DMSO and camptothecin (CPT) were used as controls. PEL cells (BC1 and BCBL1) treated with $7.5 \mathrm{nM}$ Mubritinib show a significant decrease in the total population of $\mathrm{S}$ and $\mathrm{G} 2$ cells that is not observed for BJAB or LCL352 cells. Neither cytarabine nor rapamycin showed similar selectivity for inhibition of PEL cell growth (S, G2) $\left({ }^{* *} p<0.001,{ }^{*} p<0.05\right.$; Student's $T$ Test). (B) Cell cycle profiles comparing cells (BJAB, BC1, BCBL1, and LCL352) treated with DMSO and $15 \mathrm{nM}$ Mubritinib measured by FACS flow cytometry analysis of propidium iodide staining. (C) Flow cytometry analysis of Annexin V/PI staining comparing cells (BJAB, BC1, BCBL1, LCL352) treated with DMSO and $15 \mathrm{nM}$ Mubritinib. (D) Graphs summarizing the decrease in live cell populations observed from the Annexin V/PI experiment. Camptothecin $(4 \mathrm{mM})$ and Cytarabin $(1 \mathrm{mM})$ are shown for comparison. ( ${ }^{* *} p<0.001$; Mann-Whitney). (E) ChIP-qPCR assay for LANA or IgG control in BCBL1 cells treated with Mubritinib (15 $\mathrm{nM}$ ) for 72 hrs with primers for KSHV TR, control region a, or cellular Actin. (F) Western blot control of ChIP assays showing LANA, RTA, Actin, or gH2AX in BCBL1 cells at $48 \mathrm{~h}$ after addition of DMSO (-) or Mubritinib (15 nM) (+). 
only the BCBL1 and $\mathrm{BC} 1$ mice treated with Mubritinib demonstrated a reduction in bioluminescence signals when compared to their vehicle controls.

This inhibition of PEL cell growth in vivo was further validated by measuring ascites volumes (Figure $5 \mathrm{E}$ ). Mubritinib treatment caused a significant reduction in the volumes of ascites for the BCBL1 and BC1 xenograft mice. LCL352 mice did not develop large volumes of ascites when they were treated with either vehicle or Mubritinib. Interestingly, although the total volume of ascites was reduced for the BCBL1 and $\mathrm{BC} 1$ mice upon treatment with Mubritinib (Figure 5E), the KSHV copies per $\mathrm{mL}$ of ascites for these mice remained similar to the corresponding vehicle treated controls (Figure 5F). This suggests that, at least in these in vivo experiments, Mubritinib had little effect on KSHV episome copy number. This is consistent with our previous observation that Mubritinib inhibits PEL cell growth at concentrations far lower than those that were necessary to disrupt LANA DNA binding (Figures 2 and 3).

For our in vivo experiments, we used both female and male mice (Figure 5G). And, in general, the localization of the bioluminescence signals that we observed were similar for mice of both genders. However, the male mice also developed strong signals in their testes. Interestingly, the intensities of these signals were far greater in the BCBL1 and BC1 mice than the LCL352 mice (Figure $5 \mathrm{H}$ ). Furthermore, the bioluminescence signals in the testes of the BCBL1 and $\mathrm{BC} 1$ mice were significantly reduced when the mice were treated with Mubritinib. Similarly, the bioluminescence signals in the lungs of the BCBL1 and $\mathrm{BC} 1$ mice were significantly reduced when they were treated with Mubritinib (Figure $5 \mathrm{H})$. Collectively, these data confirm that Mubritinib is a potent inhibitor of PEL cell growth in vivo and that its effects are selective when compared to EBV ${ }^{+}$LCL352 B-cells.

\section{Mubritinib does not act as a HER2/ErbB2 inhibitor in PEL cells}

Mubritinib (also known as TAK 165) was originally identified as a potent and selective inhibitor of receptor tyrosine kinase HER2/ErbB2 [37]. Subsequently, Grygielewicz et al. later demonstrated that SNU-16 gastric cancer cells that had become resistant to fibroblast growth factor receptor (FGFR) inhibitors became sensitive to treatment with Mubritinib [38]. Interestingly, the development of resistance to FGFR inhibitors in these cells was accompanied by a dramatic reduction in expression of various tyrosine kinase receptors, including HER2/ ErbB2. Therefore, it was proposed that Mubritinib acted by a mechanism that does not involve inhibition of HER2/ ErbB2. More recently, Baccelli et al. provided compelling evidence that Mubritinib selectively inhibits growth of a subset of acute myeloid leukemia (AML) cells that rely on mitochondrial oxidative phosphorylation (OXPHOS) and anaerobic glycolysis [39]. They determined that the molecular target for Mubritinib in these cells was the electron transport chain (ETC) complex I.

To clarify the mechanism of action for Mubritinib in PEL cells, we began by examining the level of HER2/ ErbB2 expression in these cells (Figure 6A). As a positive control, we also examined SNU-719 gastric cancer cells. However, we were not able to observe HER2/ErbB2 protein in PEL cells by Western blot. Similarly, we did not detect HER2/ErbB2 in lysates from KSHV BJAB cells or $\mathrm{EBV}^{+} \mathrm{LCL} 352$ cells. These results suggest that inhibition of PEL cell growth by Mubritinib may not involve inhibition of HER2/ErbB2. To further investigate this, we analyzed a series of published HER2/ErbB2 inhibitors, namely Afatinib, Dacomitinib, Emodin, and TAK-285 (Figure 6B-6E, Supplementary Table 2). However, none of these inhibitors displayed the same selectivity as Mubritinib (Figures 2E and 3G). These findings suggest that Mubritinib does not act as a HER2/ErbB2 inhibitor in PEL cells.

\section{ETC complex I inhibitors exhibit selectivities similar to Mubritinib for inhibition of PEL cell growth}

Given recent literature that suggests that Mubritinib can inhibit the electron transport chain (ETC) complex I [39], we next investigated whether published ETC complex I inhibitors exhibit similar selectivity for growth inhibition of PEL cells (Figure 7A-7C, Supplementary Table 2). Rotenone, like Mubritinib, displayed clear selectivity for inhibition of PEL cell growth at nanomolar concentrations (Figure 7B). Deguelin also exhibited partial selectivity for BCBL1 and BC1 PEL cell lines (Figure 7C). These results suggest that PEL cells are selectively sensitive to drugs targeting the ETC. More detailed analysis of the impact of basal and maximal oxygen consumption rates (OCR) and basal ATP production was measured by Seahorse analysis (Figure 7D and Supplementary Figure 3). This revealed that Mubritinib inhibits ATP production in all cells tested, but that maximal and basal OCR levels were more selectively inhibited in KSHV+ PEL cells relative to LCLs and gammaherpesvirus negative BJABs. The maximal respiratory rate is determined by several factors, including the functional capacity of the electron transport chain. Therefore, these results suggest that disruption of maximal OCR by Mubritinib is specific to KSHV+ cells and that this disruption of OXPHOS prevents the electron transport chain in $\mathrm{KSHV}+$ cells from operating at maximum capacity.

\section{Mubritinib selectively alters key metabolite levels in PEL cells}

In AML cells, inhibition of ETC complex I by Mubritinib was accompanied by specific changes in the 
A

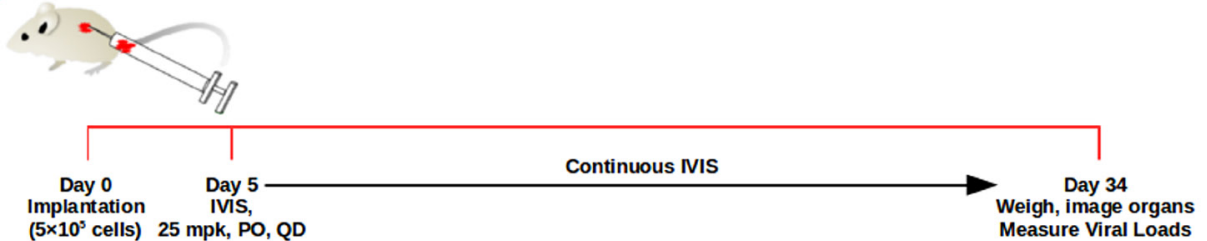

B

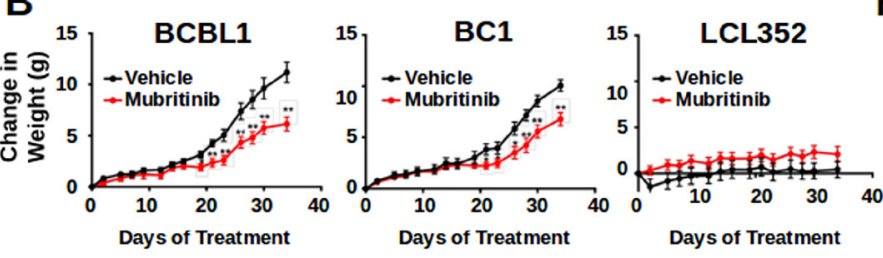

$\mathbf{F}$

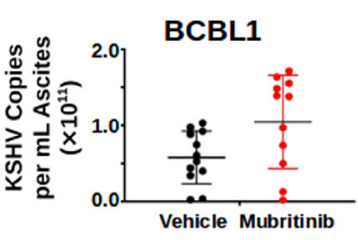

C

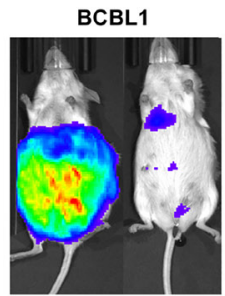

$\mathrm{BC} 1$

LCL352
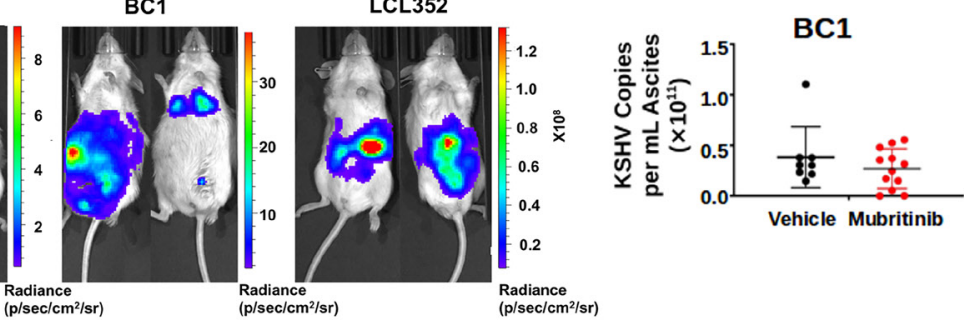

D

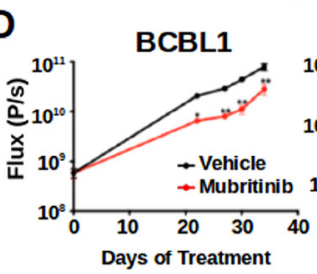

BC1

E
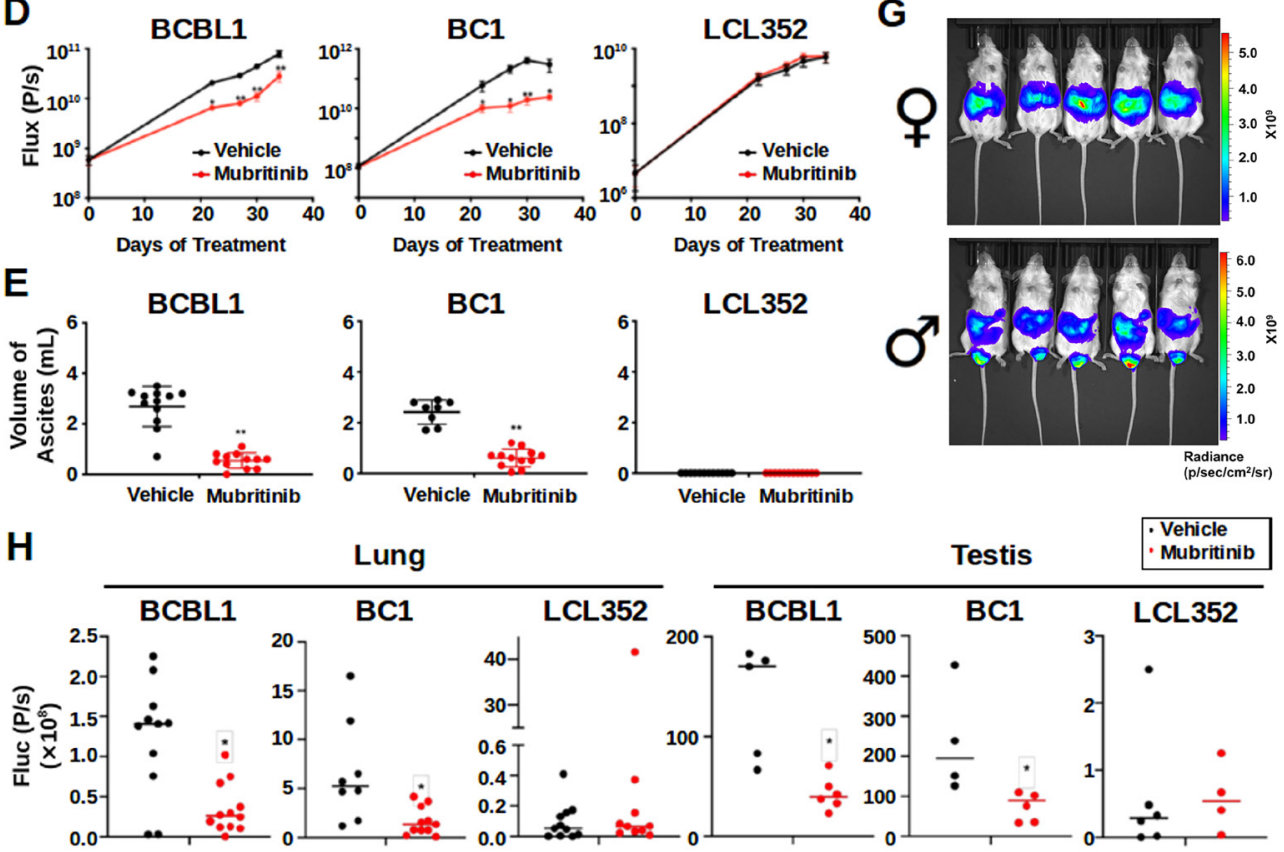

Figure 5: Mubritinib Inhibits PEL tumor growth in mouse xenografts. (A) Cartoon illustrating key features of the in vivo experiments that were performed with Mubritinib. NSG mice were implanted with $5 \times 10^{5} \mathrm{BCBL1}$, BC1, or LCL352 cells intraperitoneally on Day 0. Beginning on Day 5, the mice were treated daily with 0 (vehicle) or $25 \mathrm{mg} / \mathrm{kg}$ (mpk) Mubritinib orally. During treatment, the mice were continuously imaged using a Spectrum IVIS CT Bioluminescent Imaging System. At Day 34, the mice were sacrificed, weighed, and selected organs were collected for further analyses. (B) Graphs comparing the changes in weight that were observed in mice implanted with PEL cell lines (BCBL1 and BC1) versus mice implanted with the EBV ${ }^{+}$LCL352 B-cell line ( $p<0.05,{ }^{* *} P<0.01 ; T$ test). (C) Images comparing the bioluminescence signals that were observed in mice implanted with PEL cell lines (BCBL1 and BC1) versus mice implanted with LCL352 cells. For each pair, the left mouse was treated with vehicle and the right mouse was treated with Mubritinib. (D) Graphs showing the changes in bioluminescence flux (photons/s) over time. These results are in agreement with those observed in $(\mathrm{C})\left({ }^{* *} P<.01 ; T\right.$ test). (E) Graphs showing the volumes of ascites that were collected at the end of the in vivo experiments. ( ${ }^{* *} p<0.0001$; Mann-Whitney). Notably, animals engrafted with LCL352 cells did not develop ascites. (F) Graphs showing the number of KSHV copies per $\mathrm{mL}$ of ascites. (G) Images comparing the bioluminescence signals from female (top) and male (bottom) mice. The male mice developed a strong bioluminescence signal in their testis. (H) Graphs showing the bioluminescence flux (photons/s) in the lungs and testis in vehicle (black) vs Mubrtinib (red) ( $p<0.01$; Mann-Whitney). 
levels of various metabolites [39]. To assess the effect of Mubritinib on metabolites in PEL cells, we performed a global metabolomic analysis by mass spectrometry for a panel of B-cells, including $\mathrm{KSHV}^{+} \mathrm{BC} 1$ and $\mathrm{BCBL} 1$, and $\mathrm{KSHV}^{-} \mathrm{BJAB}$ and LCL352 (Figure 8A). As with AML cells [46], we observed that Mubritinib reduced the NAD/ $\mathrm{NADH}$ ratio in $\mathrm{KSHV}^{+} \mathrm{PEL}$ cells (Figure $8 \mathrm{~B}$ ). However, we also saw a reduction in the NAD/NADH ratio for a $\mathrm{KSHV}^{-} / \mathrm{EBV}^{-}$cell line $(\mathrm{BJAB})$ and a $\mathrm{KSHV}^{-} / \mathrm{EBV}^{+}$cell line (LCL352). Therefore, we concluded that this reduction may not explain selectivity that Mubritinib exhibits for PEL cell growth inhibition. Conversely, Mubritinib treatment produced distinctly unique responses in $\mathrm{ADP} /$ ATP (Figure 8C) and AMP/ATP (Figure 8D) ratios in PEL cells. Moreover, N-acetyl-L-aspartic acid (Figure 8E) and 5-thymidylic acid (Figure 8F) levels were also uniquely altered upon treatment with Mubritinib. Interestingly, although Baccelli et al. observed that Mubritinib treatment led to a decrease in aspartate levels in AML cells, we observed an increase in N-acetyl-L-aspartic acid in PEL cells. In contrast, we observed a decrease in N-acetylL-aspartic acid for the two $\mathrm{KSHV}^{-}$cell lines (BJAB and LCL352). These findings suggest that Mubritinib selectively alters metabolic pathways and the ETC in ways that contribute to the selective inhibition of KSHV positive PEL cell growth.

\section{DISCUSSION}

KSHV associated cancers remain an unmet medical need due to their high rates of resistance to existing chemotherapies [21]. PEL patients typically have less than $1 \mathrm{yr}$ survival time even with aggressive chemotherapy [40]. Many other drugs and natural products have been reported to inhibit PEL cell growth and tumorigenesis [35, 41-55]. However, even though KSHV is known to drive this cancer, at present there are no inhibitors that selectively and effectively target KSHV gene products.

In this work, we focused on identification of known drugs that could be readily repurposed for the treatment of PEL. We screened a small collection of known drugs from SelleckChem and identified Mubritinib as an interesting candidate based on its ability to selectively inhibit LANA DNA binding and selectively inhibit $\mathrm{KSHV}^{+}$PEL cell growth in vitro and in vivo. Remarkably, cells infected with EBV, a $\gamma$-herpesvirus that is closely related to KSHV, responded similar to other KSHV- cells when treated with Mubritinib.

Our findings indicate that Mubritinib can inhibit LANA DNA binding in a cell-based high-throughput assay (Figure 2) and in ChIP assays in PEL cells (Figure 4). Previous studies demonstrated that prolonged knockdown of the KSHV protein LANA in a PEL cell line led to the loss of KSHV episomes [56]. Similarly, CRISPR deletion of LANA led to a loss of KSHV genomes from PEL cells [33]. In these genetic disruptions, LANA depletion did not lead to cell cycle arrest or loss of viability. Although complete loss of KSHV genomes may not have been achieved, these studies raise the concern that loss of LANA and KSHV genomes may not be sufficient to block PEL tumorigenesis. Thus, it is likely that Mubritinib inhibition of PEL cell growth is due, at least in part, to activities other than LANA inhibition.

While Mubritinib was originally identified as a HER2/ErbB2 inhibitor [37], our findings suggest that its selectivity for PEL growth inhibition is dependent on its additional activity in blocking electron transport and mitochondrial metabolism [39]. Metabolic processes were recently determined to be among the top hits in a CRISPR screen for essential pathways in PEL cells [57]. In endothelial cells, KSHV is known to induce the Warburg effect, a cancer-associated metabolic shift to aerobic glycolysis $[58,59]$. Yet, suppression of glycolysis is observed during infection of mesenchymal stem cells [60]. KSHV can induce nuclear factor erythroid2-related factor 2 (NRF2) [61], a regulator of cellular resistance to oxidants, and reactive oxygen species (ROS) during endothelial cell infection [62]. Furthermore, inhibitors of ROS were shown to block mouse models of KS [62]. mTOR inhibitors induce mitochondrial apoptosis in PEL and $\mathrm{KS}$, and the mechanism may be related to disruption of oxidative phosphorylation, similar to Mubritinib and Rotenone $[63,64]$. We found that Mubritinib selectively inhibited the maximal $\mathrm{OCR}$ in $\mathrm{KSHV}^{+} \mathrm{PEL}$ relative to other B-cell lines (Figure 7D), as well as the ADP/ATP (Figure 8C) and ATP/AMP (Figure 8B) ratios. We also found Mubritinib selectively altered $\mathrm{N}$-acetyl-aspartic acid (Figure 8E) and 5-thymidylic acid (Figure 8F) abundance in $\mathrm{KSHV}^{+} \mathrm{PEL}$ cells. These findings are consistent with Mubritinib inhibition of the ETC as a mechanism of selective inhibit PEL cell growth.

In conclusion, we provide evidence that Mubritinib can inhibit LANA DNA binding and PEL cell growth. The selective inhibition of PEL cells correlated with inhibition of mitochondrial OXPHOS function. The mechanism of inhibition of LANA DNA binding by Mubritinib, as well as other RTK inhibitors, is not yet know. It is possible that dual activities of Mubritinib may enhance its selective inhibition of $\mathrm{KSHV}+$ PEL cells. In this work, we focused on the mechanism of inhibition of PEL cell growth and found a vulnerability in PEL cell mitochondrial OXPHOS function. These findings may inform development of more selective and efficacious treatments for PEL and other KSHV-associated cancers. Preclinical studies for Mubritinib in a murine model of bladder cancer were performed at doses of $10 \mathrm{mg} / \mathrm{kg}$ and $20 \mathrm{mg} / \mathrm{kg}$ administered BID (twice daily) [37]. Therefore, our study showing an effect of Mubritinib at a similar dose (25 mg/kg), administered only once daily suggests that we are well within a dose range that is consistent with studies used for other cancer indications. Moreover, the paucity of specific treatments available for PEL underscores the potential importance of our in vivo findings. 


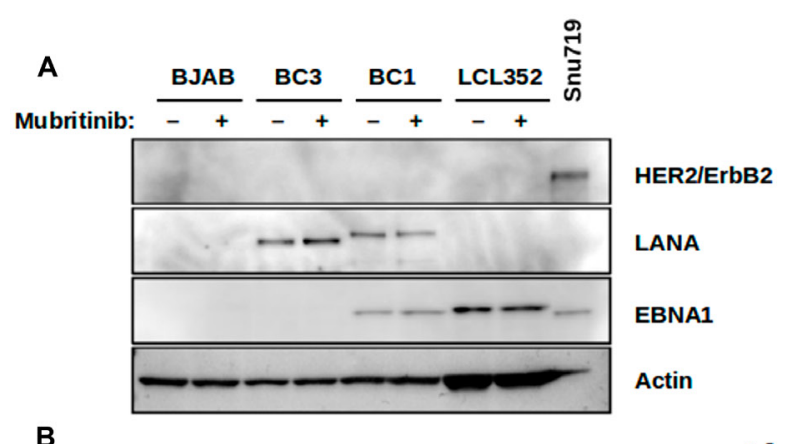

B

Afatinib

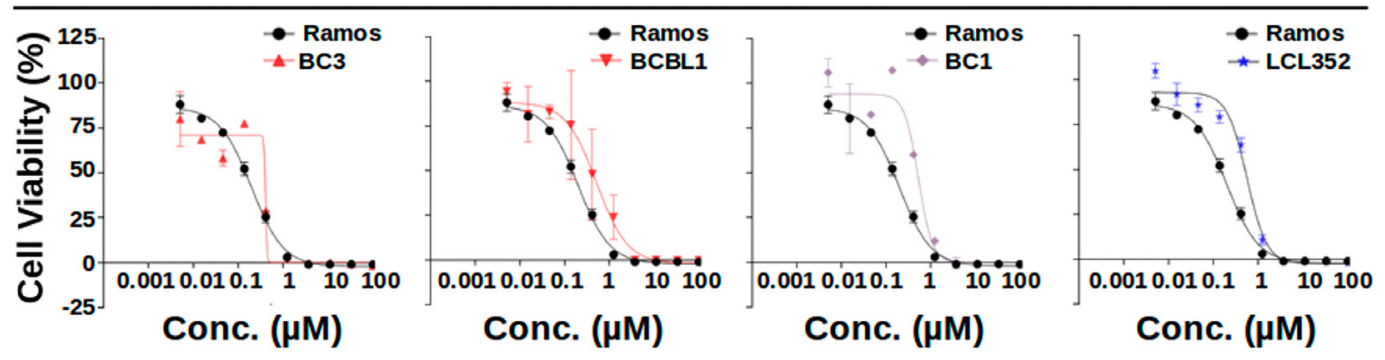

C

Dacomitinib

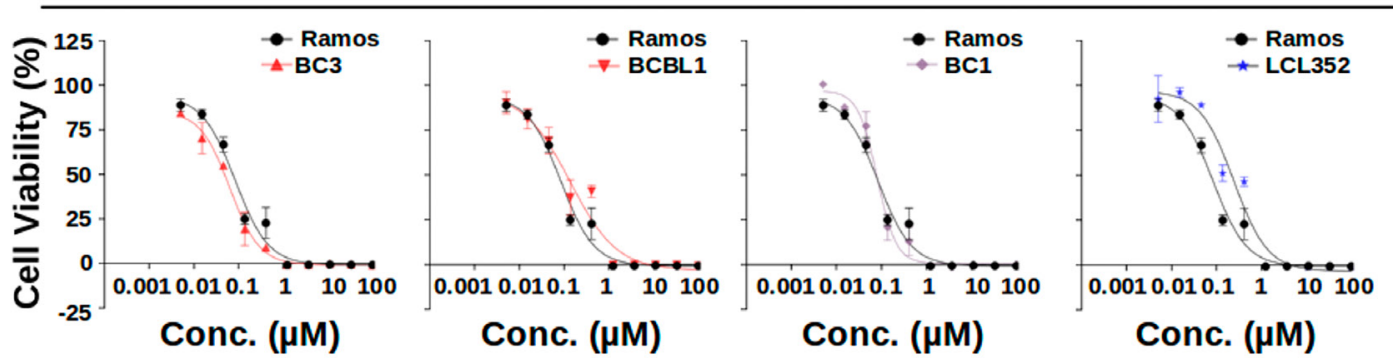

D

Emodin

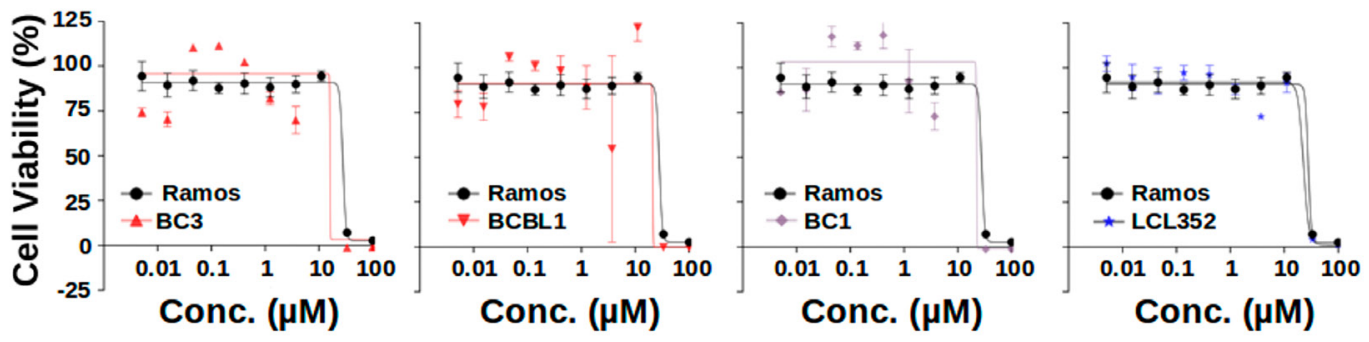

E

TAK-285

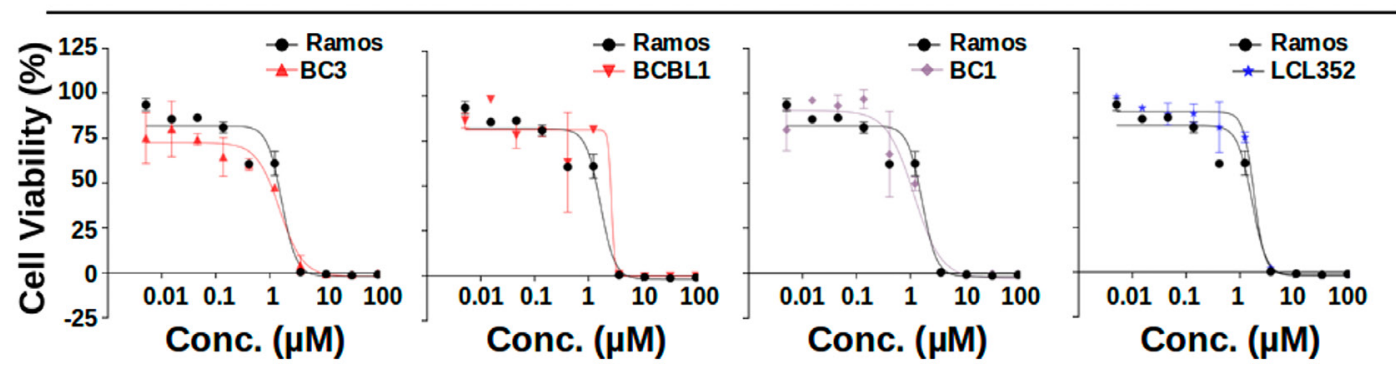

Figure 6: HER2 inhibitors are not selective for PEL cell growth inhibition. (A) Western blot demonstrating the level of HER2/ ErbB2 expression in various cell lines. SNU-719 gastric cancer cells were used as a positive control. Expression of LANA was used to confirm infection with KSHV. Expression of EBNA1 was used to confirm infection with EBV. Actin was used as a loading control. (B-E) Titration of published HER2/ErbB2 inhibitors (B) Afitinib, (C) Dacomitinib, (D) Emodin, and (E) TAK-286 against various B-cell lines. Ramos (KSHV $\left.\mathrm{EBV}^{-}\right)$data are shown for reference in each graph. None of these HER2/ErbB2 inhibitors display the same selectivity for PEL cell growth inhibition as Mubritinib shown in Figure 3E. 

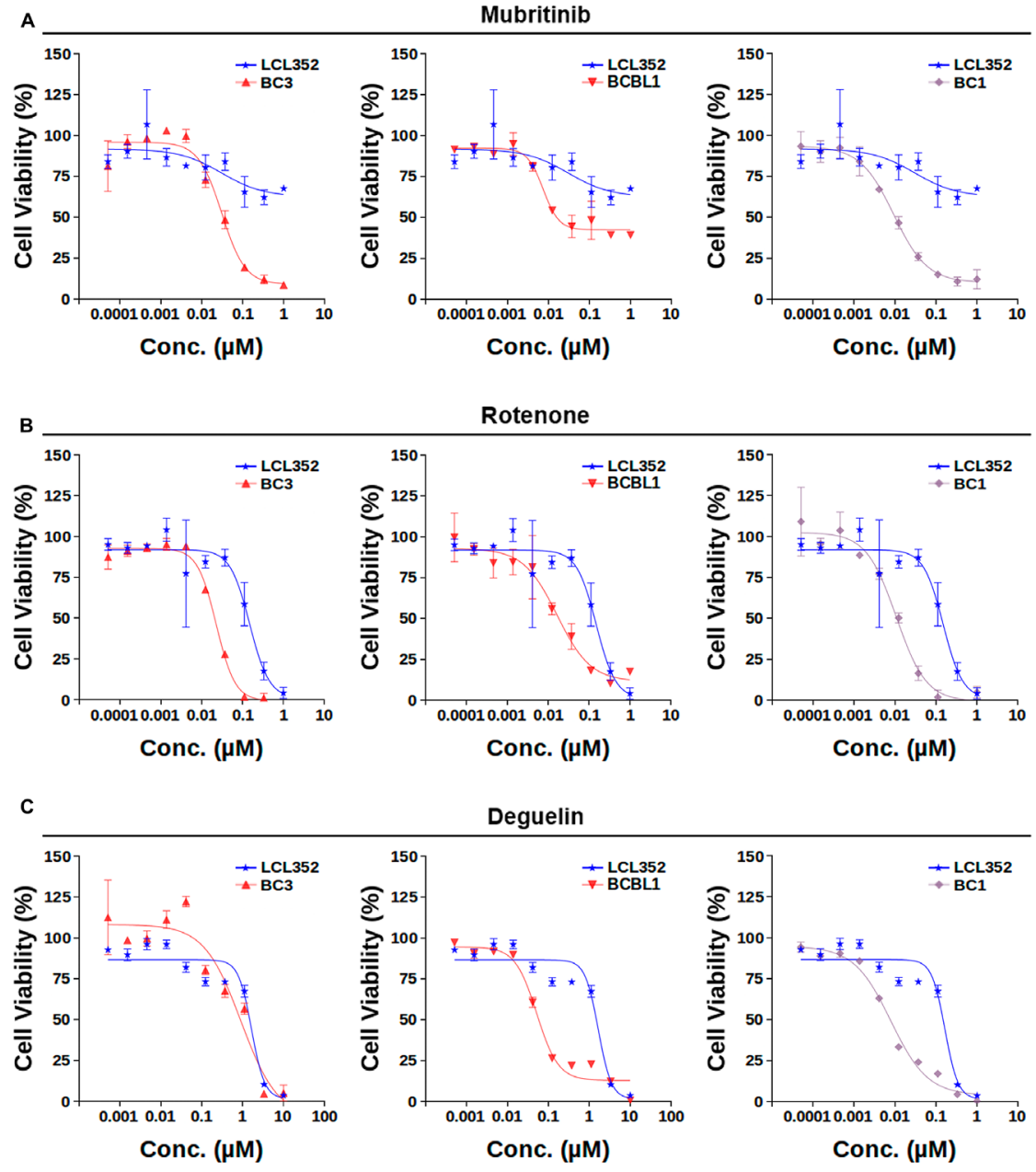

D

BC1

BCBL1

BJAB

LCL352
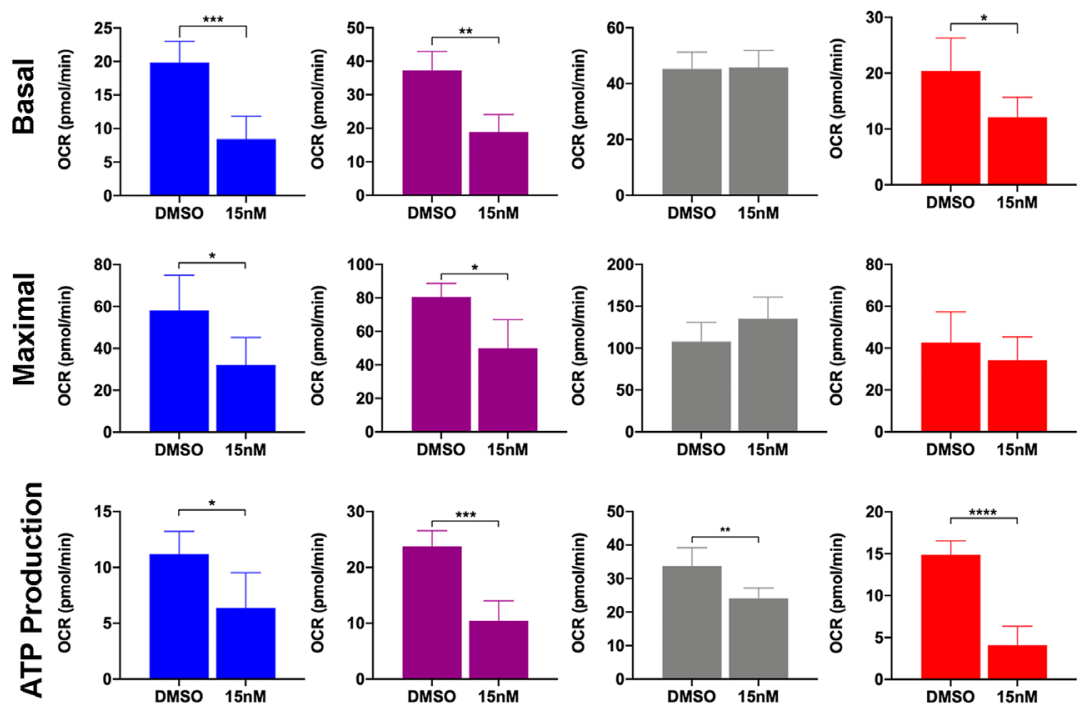

Figure 7: Electron transport chain (ETC) complex I inhibitors exhibit selectivities similar to Mubritinib for inhibition of PEL cell growth. (A) Titration of Mubritinib against various B-cell lines. EBV ${ }^{+}$(LCL352) data (blue) are shown for reference in each graph. $\mathrm{KSHV}^{+}$PEL cell lines $(\mathrm{BC} 3, \mathrm{BCBL} 1$, and $\mathrm{BC} 1)$ respond more strongly to Mubritinib at nanomolar concentrations. (B) Titration of Rotenone, an ETC complex I inhibitor, against the same B-cell lines shown in (A). (C) Titration of Deguelin, an ETC complex I inhibitor, against the same B-cell lines shown in (A). (D) Effects of Mubritinib on basal and maximal oxygen consumption rates (OCR) as well as ATP production for each cell line. $\left({ }^{* * *} p<0.0001,{ }^{* *} p<0.001,{ }^{*} p>0.05 ; T\right.$-test $)$. 


\section{MATERIALS AND METHODS}

\section{Chemicals}

The SelleckChem library from the Molecular Screening Facility at the Wistar Institute was used for initial screens. However, once hits were identified, additional material was purchased for further testing. Mubritinib (S2216), Carmofur (S1289), Erlotinib HCl (S1023), NSC-207895 (S2678), OSI-420 (S2205), Emodin

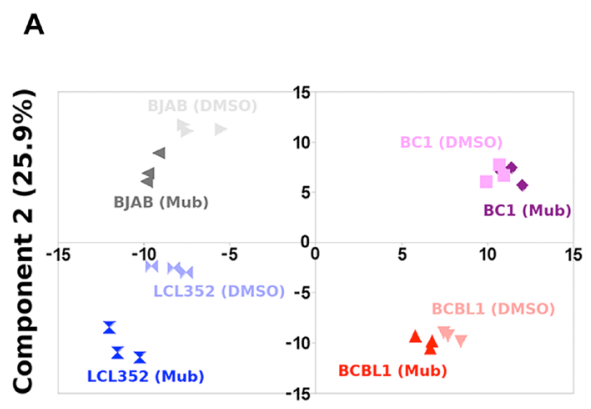

Component 1 (30.7\%)
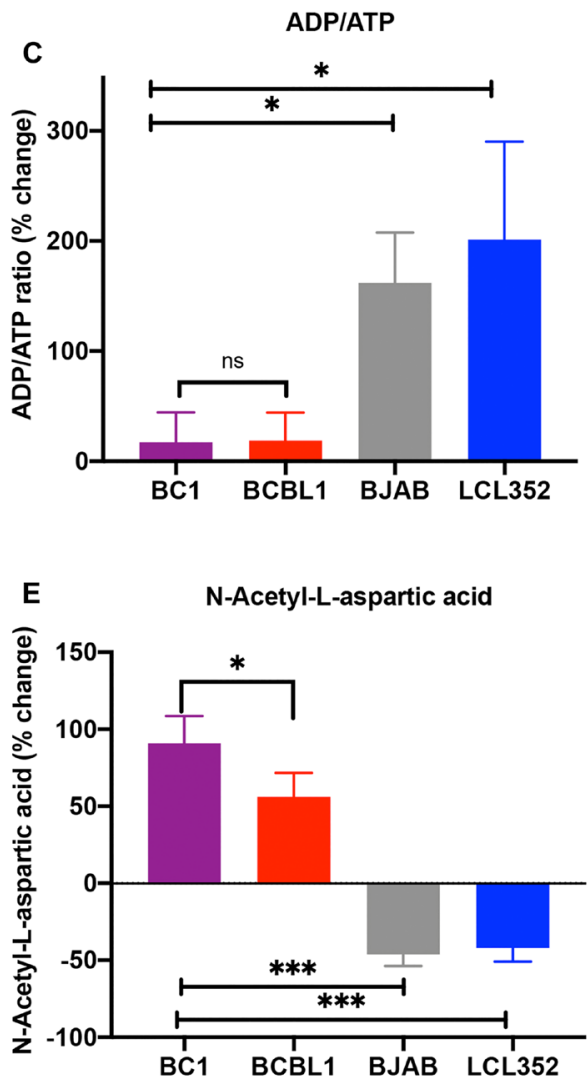

(S2295), TAK-285 (S2784), and Deguelin (S8132) were purchased from SelleckChem. Linifanib (M15676), Regorafenib (R16040), and Afatinib (G-7208) were purchased from AChemBlock. Adrucil (228440010) and Cytarabine (449561000) were purchased from Acros Organics. Lenalidomide (6305/100) and Dopamine $\mathrm{HCl}(3548 / 50)$ were purchased from Tocris Bioscience. Dacomitinib (PZ0330) and Rotenone (R8875) were purchased from Sigma. Rapamycin (J62473) was purchased from Alfa Aesar.
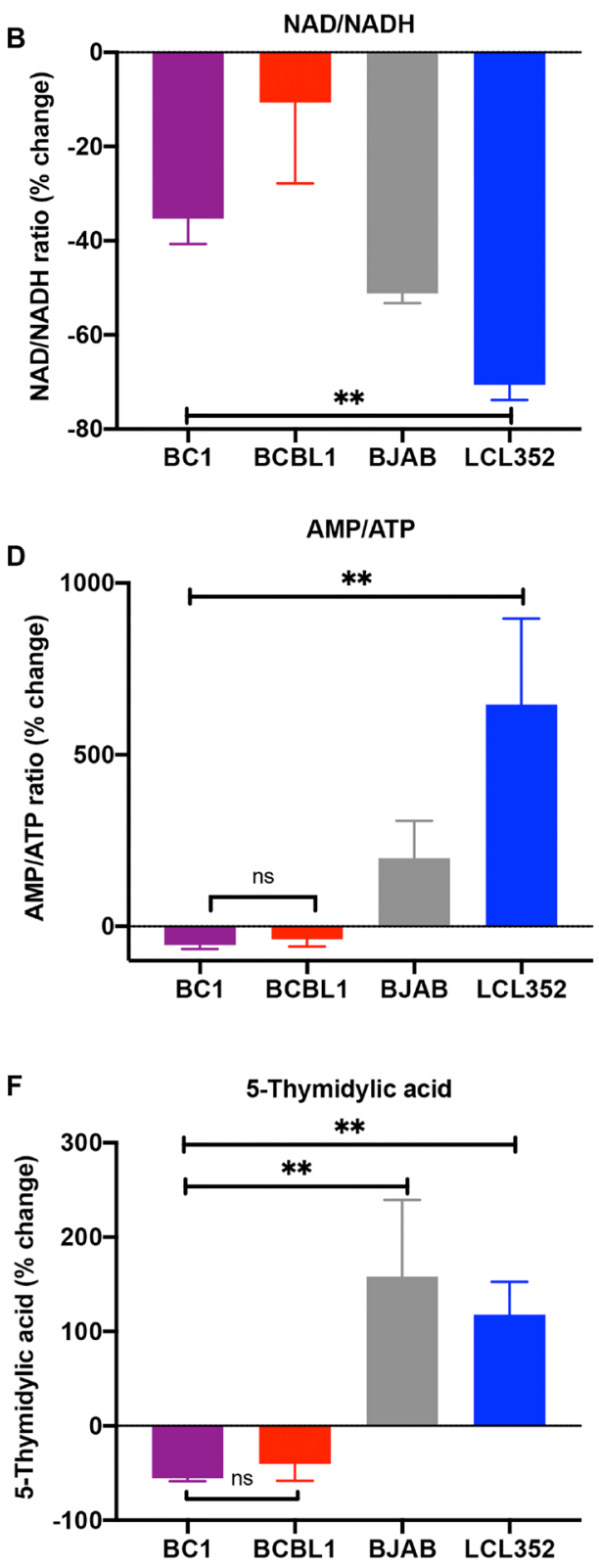

Figure 8: Mubritinib selectively alters key metabolite levels and impairs the electron transport chain in PEL cells. (A) Principle component analysis (PCA) for the metabolomics data. These results confirm that sample replicates cluster together based on cell line (BJAB, LCL352, BC1, BCBL1) and treatment condition (DMSO, Mubritinib). (B) Percent change in NAD/NADH ratios. (C) Percent change in AMP/ATP ratio observed upon treatment with Mubritinib (relative to DMSO treatment) for each cell line. (D) Percent change in ADP/ATP ratios. (E) Percent change in N-acetyl-L-apartic acid levels (relative levels determined by normalized peak area). (F) Percent changes in 5-thymidylic acid levels (relative levels determined by normalized peak area). ${ }^{* * *} p<0.001,{ }^{* *} p<0.01,{ }^{*} p>0.05$; One-WayAnova, post-hoc analysis by Tukey test). 


\section{Antibodies}

The following antibodies were used for Western blot analysis: rabbit $\beta$-tubulin (Cell Signaling Technology, 2146S), goat anti-rabbit IgG (H+L)-HRP Conjugate (Bio-Rad, 1706515), ANTI-FLAG M2-Peroxidase (Sigma Aldrich, A8592), Apoptosis Western Blot Cocktail (abcam, ab136812), rat anti-LANA (Advanced Biotechnologies Inc., 13210), mouse anti-EBNA1 (Biorad), rabbit anti-HER2 (D8F12) (Cell Signaling), mouse monoclonal anti-actin-HRP (Sigma).

\section{Plasmids}

The Gaussia luciferase reporter plasmid was generated by removing the firefly luciferase sequence from the pGL4.31 plasmid using FseI and HindIII-HF enzymes (New England Biolabs) and replacing it with the Gaussia luciferase sequence. Then, two complementary oligonucleotides containing an AflII restriction site were inserted upstream of the MLP promoter using EcoRIHF and NheI enzymes (New England Biolabs). Finally, complementary oligonucleotides containing the three LANA DNA binding sites (LBS2-LBS1-LBS3) were inserted upstream of the MLP promoter using AflII and NheI enzymes (New England Biolabs). The RMCE-HILO donor plasmid was generated by digesting the pEM791 plasmid [65] with AgeI-HF and BsrGI-HF enzymes (New England Biolabs) and inserting two PCR amplified sequences containing the FLAG tag fused to the VP16 activation domain and the LANA DNA-binding domain by Gibson Assembly (New England Biolabs).

\section{Cell lines}

The RMCE-HILO HEK293T acceptor cells were obtained from Eugene V. Makeyev (Nanyang Technological University, Singapore) and maintained in Dulbecco's modified Eagle's medium (Gibco BRL) with $10 \%$ FetalPlex Serum Complex (Gemini Bio) and penicillin and streptomycin $(50 \mathrm{U} / \mathrm{ml})$. Ramos cells (uninfected Burkitt Lymphoma from ATCC CRL-1596) and $\mathrm{BJAB}$ (uninfected B cell lymphoma) cells were obtained from ATCC and maintained in RPMI medium (Gibco BRL) containing 10\% heat-inactivated fetal bovine serum and penicillin and streptomycin $(50 \mathrm{U} / \mathrm{ml})$. $\mathrm{KSHV}^{+}$ single positive PEL cells (BCBL1 and $\mathrm{BC} 3$ ) and double positive KSHV and EBV infected PEL cells (BC1) were provided by Yan Yuan (University of Pennsylvania) and maintained in RPMI medium (Gibco BRL) containing $10 \%$ heat-inactivated fetal bovine serum and penicillin and streptomycin $(50 \mathrm{U} / \mathrm{ml})$.

\section{Gaussia luciferase screens}

On the first day, 4-5 $\times 10^{6}$ HEK293T acceptor cells were seeded in a $10-\mathrm{cm}$ plate. The following day, the media was replaced with antibiotic-free media and the cells were transfected with $3 \mu \mathrm{g}$ reporter plasmid using Lipofectamine 2000 (Invitrogen) at a ratio of 1:3 (plasmid: transfection reagent). Approximately 24 hours after transfection, the cells were trypsinized, counted, and diluted to 150,000 cells $/ \mathrm{mL}$ using Dulbecco's modified Eagle's medium (Gibco BRL) with 2.5\% FetalPlex Serum Complex (Gemini Bio) and penicillin and streptomycin $(50 \mathrm{U} / \mathrm{ml})$. These diluted cells were separated into two aliquots (-dox and + dox) and doxycycline was added to one of the aliquots to a final concentration of $0.125 \mu \mathrm{g} /$ $\mathrm{mL}$. Then, $40 \mu \mathrm{L}$ of cells were seeded into each well of a 384-well plate. Control wells were treated with DMSO at a final concentration of $0.125 \%$. The remaining wells were treated with drugs from the SelleckChem library (dissolved in DMSO) at a final concentration of $12.5 \mu \mathrm{M}$. Approximately 24 hours after addition of the drugs, the Gaussia luciferase signal for each well was measured (BioLux Gaussia Luciferase Assay Kit, New England Biolabs) using an Envision Plate Reader. Then, the cells were incubated with resazurin (Sigma) at a final concentration of $50 \mu \mathrm{M}$ for $\sim 4$ hours and the cell viability was measured using an Envision Plate Reader. The \% luciferase signal for each drug was calculated relative to the -dox value $(0 \%)$ and the + dox value $(100 \%)$. The $\%$ cell viability for each drug was calculated relative to complete inhibition of cell viability $(0 \%)$ and treatment with DMSO (100\%).

\section{B-cell viability screens}

$\mathrm{BC} 3$ and Ramos cells were counted and diluted to 100,000 cells $/ \mathrm{mL}$ and 150,000 cells $/ \mathrm{mL}$, respectively, with RPMI medium (Gibco BRL) containing 2.5\% heat-inactivated fetal bovine serum and penicillin and streptomycin. Each cell line was separated into two aliquots (-puro and +puro) and puromycin (SigmaAldrich) was added to one of the aliquots. Then, 50 $\mu \mathrm{L}$ of cells were seeded into each well of a 384-well plate. Control wells were treated with DMSO at a final concentration of $0.1 \%$. The remaining wells were treated with drugs from the SelleckChem library (dissolved in DMSO) at a final concentration of $10 \mu \mathrm{M}$. Approximately 72 hours after addition of the drugs, the cells were incubated with resazurin (Sigma) at a final concentration of $50 \mu \mathrm{M}$ for $\sim 4$ hours and the cell viability was measured using an Envision Plate Reader. The \% cell viability for each drug was calculated relative to the +puro value $(0 \%)$ and treatment with DMSO (100\%).

\section{B-cell hit titrations}

Cells were diluted with RPMI containing 2.5\% heat-inactivated fetal bovine serum and penicillin and streptomycin as follows: Ramos $(120,000$ cells $/ \mathrm{mL}), \mathrm{BC} 3$ $(160,000$ cells $/ \mathrm{mL})$, BCBL1 $(80,000$ cell $/ \mathrm{mL})$, BJAB 
(12,000 cells/mL), BC1 (100,000 cells/mL), LCL352 $(40,000$ cells $/ \mathrm{mL})$. Then, cells were seeded at a final volume of $50 \mu \mathrm{L}$ into a 384-well plate and treated with drugs dissolved in DMSO. The final concentration in the well was $0.1 \%$. Wells treated with DMSO or puromycin (+puro) were used as controls. Approximately 72 hours after addition of the drugs, the cells were incubated with resazurin (Sigma) at a final concentration of $50 \mu \mathrm{M}$ for $\sim 4$ hours and the cell viability was measured using an Envision Plate Reader. The \% cell viability for each drug was calculated relative to the + puro value $(0 \%)$ and treatment with DMSO (100\%).

\section{Analysis of cell cycle kinetics}

$\mathrm{BC} 1\left(\mathrm{KSHV}^{+}, \mathrm{EBV}^{+}\right), \mathrm{BCBL} 1\left(\mathrm{KSHV}^{+}, \mathrm{EBV}^{-}\right)$, and LCL352 (KSHV-, EBV $\left.{ }^{+}\right)$cells were seeded in 6-well plates and exposed to camptothecin $(4 \mu \mathrm{M})$, rapamycin $(40 \mathrm{nM})$, cytarabine $(1 \mu \mathrm{M})$, or Mubritinib (15 $\mathrm{nM}$ and $7.5 \mathrm{nM})$ in biological triplicates per each condition. After $72 \mathrm{~h}$, cells were permeabilized with cold, $70 \%$ ethanol and resuspended in PBS containing PI (10 mg/mL) and RNAse A solution $(100 \mu \mathrm{g} / \mathrm{mL})$. Flow cytometry was performed on a BD-LSR II (BD Biosciences; Bedford, MA, USA) and data were analyzed using FloJo software (Ashland, OR, USA).

\section{Annexin V/PI binding assay}

An AnnexinV-FITC apoptosis detection kit (Abcam; Cambridge, UK) was used to confirm the effect of Mubritinib and other compounds of interest on cell viability. $\mathrm{BC} 1\left(\mathrm{KSHV}^{+}, \mathrm{EBV}^{+}\right), \mathrm{BCBL} 1\left(\mathrm{KSHV}^{+}, \mathrm{EBV}^{-}\right)$, and LCL352 (KSHV, $\left.\mathrm{EBV}^{+}\right)$cells were seeded in 6-well plates and exposed to camptothecin $(4 \mu \mathrm{M})$, rapamycin $(40 \mathrm{nM})$, vargatef (40 nM), AST-1306 (10 nM), cytarabine $(1 \mu \mathrm{M})$, or Mubritinib (15 nM and $7.5 \mathrm{nM}$ ) in biological triplicates per each condition. After $72 \mathrm{~h}$, the percentages of live and apoptotic cells were analyzed after double staining the cells with FITC conjugated Annexin V and propidium iodide (PI). Flow cytometry was performed on a BD-LSR II (BD Biosciences; Bedford, MA, USA) and data were analyzed using FloJo software (Ashland, OR, USA).

\section{Ethics statement}

All animal experiments were conducted under the Wistar Institute's approved Institutional Animal Care and Use Committee Protocol \#201158 in accordance with the Committee for the Purpose of Control and Supervision of Experiments on Animals guidelines for animal experimentation. All mice in this study were managed in accordance with the NIH Office of Laboratory Animal Welfare: "PHS Policy on the Humane Care and Use of Research Animals"; the recommendations of the American Association for Accreditation of Laboratory Animal Care (AAALAC).

\section{Mice}

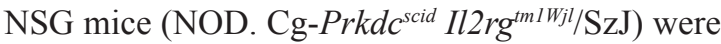
bred in-house at The Wistar Institute under protocol \#112092. Mice were enrolled at 6-8 weeks of age and housed in micro-isolator cages in a designated, specific pathogen-free facility where they were fed sterile food and water ad libitum. Mice were euthanized via $\mathrm{CO}_{2}$ administration according to AALAC euthanasia guidelines.

\section{Tumor implantation, grouping, and equalization}

Mice (six males and six females per treatment group) were engrafted with a cell suspension ( $>98 \%$ viability) of $5 \times 10^{5} \mathrm{BC} 1$-mCherry/eLUC (KSHV+, $\left.\mathrm{EBV}^{+}\right), 5 \times 10^{5} \mathrm{BCBL} 1$-mcherry/eLUC $\left(\mathrm{KSHV}^{+}, \mathrm{EB}\right.$ $\left.\mathrm{V}^{-}\right)$, or $5 \times 10^{5}$ LCL352- mCherry/eLUC $\left(\mathrm{KSHV}^{-}, \mathrm{EBV}^{+}\right)$ cells resuspended in $1 \times \mathrm{PBS}, \mathrm{pH} 7.4$ and maintained on ice. Animals were weighed three-times per week and monitored daily. The Spectrum IVIS CT Bioluminescent Imaging System (Perkin-Elmer; Waltham, MA) was used to randomize mice into groups of 12 per treatment (six males and six females) on day five so ensure the average Flux (Photons/sec) was equivalent across groups and to monitor cell growth and metastasis throughout the study. For imaging studies, mice were injected with D-luciferin (Gold Biotechnology), i.p. at a dose of $7.5 \mathrm{mg} / \mathrm{kg}$ in a dose volume of $10 \mathrm{ml} / \mathrm{kg}$ body weight 15 minutes prior to imaging; this was the optimal interval between luciferin injection and bioluminescent imaging as determined by an initial kinetic curve for these cell lines in mice. Mice were anesthetized using isoflurane prior to imaging. Total body Flux (Photons/Sec) was quantitated throughout the study using the IVIS imaging software. Flux measurements for the liver, spleen, kidneys, heart, lungs, and testis were obtained postmortem at the terminal timepoint on day 34 .

\section{Mubritinib formulation and treatment schedule}

Mubritinib was weighed and vehicle was added immediately prior to dose. The vehicle was $10 \%$ DMSO, $2.5 \%$ Tween $^{\circledR} 80+97.5 \%$ of $0.5 \%$ Carboxy Methyl Cellulose (CMC). The vehicle control comprised formulation reagents without compound. Mubritinib was administered q. d., p. o. at $25 \mathrm{mg} / \mathrm{kg}$ in a dose volume of $10 \mathrm{ml} / \mathrm{kg}$ body weight.

\section{Quantitative PCR for ascites viral load}

Viral loads were quantified with a standard curve using quantitative PCR. Standards were prepared using a full-length KSHV genome bacterial artificial chromosome, BAC16 (1). Using a determined DNA concentration and size of the KSHV genome $(138 \mathrm{Kbp})$, we calculated BAC16 copy number per microliter. We 
then prepared a series of six 10-fold dilution standards of known concentration and copy number from the isolated BAC16 DNA stock. Total DNA was extracted from ascites fluid using the DNeasy Blood and Tissue kit (Qiagen; Germantown, MD, USA). All DNA samples were plated in triplicate on a 384 well plate. Each well contained $5 \mu \mathrm{l}$ of sample and $10 \mu \mathrm{l}$ of master mix. The master mix in each well included $7.5 \mu$ of SYBR green, $0.15 \mu \mathrm{l}$ of each $10 \mu \mathrm{mol}$ primer for ORF50 (Sequences: 5'-CCCGCCCAGAAACCAGTAG-3' and 5'-TGCGGAGTAAGGTTGACTTTTTAA-3'), and 2.2 $\mu \mathrm{l}$ of ddH20. Samples were amplified using Applied Biosystems 7900HT Fast Real Time PCR machine. Each cycle consisted of four stages: 1) $50^{\circ} \mathrm{C}$ for 2 minutes; 2) $95^{\circ} \mathrm{C}$ for 10 minutes; 3) $95^{\circ} \mathrm{C}$ for 15 seconds followed by $60^{\circ} \mathrm{C}$ for 1 minute; 4) $95^{\circ} \mathrm{C}$ for 15 seconds followed by $60^{\circ} \mathrm{C}$ for 1 minutes. $\mathrm{Ct}$ values were plotted against the logarithm of copy number for BAC16 standards to generate a regression line $\left(\mathrm{R}^{2}>0.99\right)$. The regression equation was used to determine copy numbers for each sample.

\section{Quantitative PCR for KSHV mRNA expression}

RNA was extracted from $\mathrm{BC} 1$ and $\mathrm{BCBL} 1$ cells treated with Mubritinib (15 nM), TPA (20 ng/ml)/NaB (2 $\mathrm{mM}$ ), or DMSO control. Superscript IV RT (Invitrogen) was used to synthesize cDNA. qPCR was performed as above. Primers used for qPCR of KSHV genes include: ORF50 (5'-CAGCGTCCACTCCTGCAA-3' and 5'-CCGGTGTTCTCTGCGACAA-3'); ORF45 (5'-CGT CCGGAGAGTTGGAACTG' and 5'-GCGATCGTC GACCTGACAT-3'); PAN (5'-CGGTGTTTTGGCT GGGTTT' and 5'-AAACCTTGCCGTCTGGTCACT-3'); LANA (5'-GAGTCTGGTGACGACTTGGAG3' and 5'-A GGAAGGCCAGACTCTTCAAC-3'); ORF72 (5'-CA TTGCCCGCCTCTATTATCA and 5'-ATGACGTTGGC AGGAACCA-3'); ORF71 (5'-TGCGACCTGCACG AAACA and 5'-GGAGGAGGGCAGGTTAACGT-3'). GUSB was used as a cellular control: GUSB (5'-CGC CCTGCCTATCTGTATTCand 5'-TCCCCACAGGGAG TGTGTAG-3').

\section{Metabolomics}

Cells were diluted with RPMI containing 2.5\% heat-inactivated fetal bovine serum and penicillin and streptomycin as follows: BCBL1 $(80,000$ cell/mL), BJAB (24,000 cells/mL), BC1 (115,000 cells/mL), LCL352 $(60,000$ cells $/ \mathrm{mL})$. Then, the cells were seeded in a $15-\mathrm{cm}$ dish and treated with $15 \mathrm{nM}$ Mubritinib at a final DMSO concentration of $0.1 \%$. As a control, growth media was treated with DMSO at a final concentration of $0.1 \%$. Approximately 72 hours after treatment, the cells were collected by centrifugation, washed twice with ice cold PBS, and resuspended in extraction buffer containing $80 \%$
$\mathrm{MeOH}, 20 \%$ water, and heavy-labeled internal standards. Then, the samples were briefly vortexed and centrifuged at $13,000 \mathrm{rpm}$ for $15 \mathrm{~min}$ and $4^{\circ} \mathrm{C}$ in a microcentrifuge. Supernatants were collected for analysis. The pellets were briefly dried and used to determine protein concentration for normalization. The metabolomics analysis was performed at The Wistar Institute Proteomics and Metabolomics Shared Resource on a Thermo Q-Exactive HF-X mass spectrometer. Each experiment was performed in triplicate.

\section{Mitochondrial function}

We assessed the effect of Mubritinib (15 nM) treatment on mitochondrial function using the Seahorse XF Cell Mito Stress Kit assay (Agilent, UK). Briefly, BJAB, BC1, BCBL1, and LCL352 cells were treated overnight with Mubritnib (15 nM) or DMSO control. The day of the assay, $2 \times 10^{5}$ live cells per well were plated in a 96 well plate in Seahorse medium (Agilent, UK)

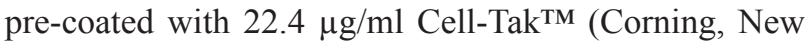
York). The Seahorse assay was run per kit instructions. Concentrations of drugs to modulate respiration were optimized for each cell line based on empirical determination of optimal oxygen consumption rate (OCR) curves. For BJAB, BC1, and BCBL1 we used $2 \mu \mathrm{M}$ oligomycin, $1 \mu \mathrm{M}$ carbonyl cyanide-4 (trifluoromethoxy) phenylhydrazone (FCCP), $1 \mu \mathrm{M}$ rotenone and antimycin A. In LCL352, the drug concentrations were reduced to used $1 \mu \mathrm{M}$ oligomycin, $0.5 \mu \mathrm{M}$ carbonyl cyanide- 4 (trifluoromethoxy) phenylhydrazone (FCCP), and 0.51 $\mu \mathrm{M}$ rotenone and antimycin $\mathrm{A}$.

\section{Abbreviations}

AAALAC: American Association for Accreditation of Laboratory Animal Care; ADP: adenosine diphosphate; AML: adult acute myeloid leukemia; AMP: adenosine monophosphate; ATP: adenosine triphosphate; ChIP: chromatin immunoprecipitation assay; CRISPR: clustered regularly interspaced short palindromic repeats; CPT: camptothecin; CYT: Cytarabine; DBD: deoxyribonucleic acid binding domain; DMSO: dimethyl sulfoxide; DNA: deoxyribonucleic acid; EBNA1: Epstein Barr virus Nuclear Antigen 1; EBV: Epstein Barr virus; ETC: electron transport chain; ErbB2: erb-b2 receptor tyrosine kinase 2; FCCP: phenylhydrazone; FITC: fluorescein isothiocyanate; HER2: human epidermal growth factor receptor 2; HHV-8: human herpesvirus 8; HRP: horseradish peroxidase; hTERT: human telomerase reverse transcriptase; IVIS CT: in vivo imaging system computerized tomography; KS: Kaposi's sarcoma; KSHV: Kaposi's sarcoma associated herpesvirus; KSHV TR: Kaposi's sarcoma associated herpesvirus terminal repeats; LANA: latency-associated Nuclear Antigen; OXPHOS: oxidative phosphorylation; MCD: multicentric Castleman's disease; MeOH: methanol; MLP: 
adenovirus major late promoter; MUB: Mubritinib; NAD: nicotinamide adenine dinucleotide; NADH: nicotinamide adenine dinucleotide; NRF2: nuclear factor erythroid2related factor 2; OCR: oxygen consumption rate; ORF: open reading frame; PAN: polyadenylated nuclear RNA; PCR: polymerase chain reaction; PEL: primary effusion lymphoma; PI: propidium iodide; PO: by mouth, orally; QD: quaque die, once daily; qPCR: quantitative PCR; RAP: rapamycin; Rb: retinoblastoma tumor suppressor; RMCEHILO: high-efficiency and low-background recombinationmediated cassette exchange technology; RPMI: Roswell Park Memorial Institute medium; ROCK: Rho-associated protein kinase; ROS: reactive oxygen species; RTK: receptor tyrosine kinase inhibitor; RTA: replication and transcription activator.

\section{Author contributions}

AC performed experiments, conceptualization, data curation, manuscript preparation. SSS performed experiments, conceptualization, data curation, and manuscript preparation. A.D.L, Z.D, D.M.F, E.M.A., Y.Z. O.V., F.L., J.C.L. performed experiments. MEM conceptualization and resources. PML conceptualization, resources, manuscript preparation.

\section{ACKNOWLEDGMENTS}

We thank Isabela Oliva and Joel Cassel at the Wistar Molecular Screening Core, Hsin Yao Tang and Aaron Goldman in the Proteomics and Metabolomics Core, and Sabrina Murdova.

\section{CONFLICTS OF INTEREST}

PML is a founder and consultant for Vironika, LLC, and has consulted for Glaxo-Smith-Kline. All other authors have no COI declarations.

\section{FUNDING}

This work was supported by research grants from NIH, R01 CA186775, R01 CA117830 (PML), PO1 CA174439 (Robertson), NCI T32 Training Grant CA009171 to (to AC), and the Wistar Institute NCI Cancer Center grant P30 CA0101815-50 (Altieri), S10 OD021669 for the IVIS Spectrum CT, S10OD023658 for the Seahorse XFe96, and S10 OD023586 for Thermo Q-Exactive HF-X mass spectrometer.

\section{REFERENCES}

1. Cesarman E, Damania B, Krown SE, Martin J, Bower M, Whitby D. Kaposi sarcoma. Nat Rev Dis Primers. 2019; 5:9. https://doi.org/10.1038/s41572-019-0060-9. [ [PubMed]
2. Ganem D. KSHV-induced oncogenesis. In: Arvin A, Campadelli-Fiume G, Mocarski E, Moore PS, Roizman B, Whitley R, Yamanishi K, editors. Human Herpesviruses: Biology. Cambridge: Therapy and Immunoprophylaxis; 2007.

3. Chang Y, Moore P. Twenty years of KSHV. Viruses. 2014; 6:4258-64. https://doi.org/10.3390/v6114258. [PubMed]

4. Cesarman E, Chang Y, Moore PS, Said JW, Knowles DM. Kaposi's sarcoma-associated herpesvirus-like DNA sequences in AIDS-related body-cavity-based lymphomas. N Engl J Med. 1995; 332:1186-91. https://doi.org/10.1056/ NEJM199505043321802. [PubMed]

5. Soulier J, Grollet L, Oksenhendler E, Cacoub P, CazalsHatem D, Babinet P, d'Agay MF, Clauvel JP, Raphael M, Degos L, Sigaux F. Kaposi's sarcoma-associated herpesvirus-like DNA sequences in multicentric Castleman's disease. Blood. 1995; 86:1276-80. [PubMed]

6. Chang Y, Cesarman E, Pessin MS, Lee F, Culpepper J, Knowles DM, Moore PS. Identification of herpesviruslike DNA sequences in AIDS-associated Kaposi's sarcoma. Science. 1994; 266:1865-9. https://doi.org/10.1126/ science.7997879. [PubMed]

7. Mutlu AD, Cavallin LE, Vincent L, Chiozzini C, Eroles P, Duran EM, Asgari Z, Hooper AT, La Perle KM, Hilsher C, Gao SJ, Dittmer DP, Rafii S, Mesri EA. In vivo-restricted and reversible malignancy induced by human herpesvirus- 8 KSHV: a cell and animal model of virally induced Kaposi's sarcoma. Cancer Cell. 2007; 11:245-58. https://doi. org/10.1016/i.ccr.2007.01.015. [PubMed]

8. Guo HG, Sadowska M, Reid W, Tschachler E, Hayward G, Reitz M. Kaposi's sarcoma-like tumors in a human herpesvirus 8 ORF74 transgenic mouse. J Virol. 2003;

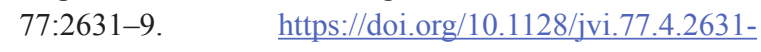
2639.2003. [PubMed]

9. Sin SH, Kim Y, Eason A, Dittmer DP. KSHV Latency Locus Cooperates with Myc to Drive Lymphoma in Mice. PLoS Pathog. 2015; 11:e1005135. https://doi.org/10.1371/journal. ppat.1005135. [PubMed]

10. Ballon G, Chen K, Perez R, Tam W, Cesarman E. Kaposi sarcoma herpesvirus (KSHV) vFLIP oncoprotein induces $\mathrm{B}$ cell transdifferentiation and tumorigenesis in mice. $\mathrm{J}$ Clin Invest. 2011; 121:1141-53. https://doi.org/10.1172/ $\underline{\mathrm{JCI} 44417}$. [PubMed]

11. Ballestas ME, Chatis PA, Kaye KM. Efficient persistence of extrachromosomal KSHV DNA mediated by latencyassociated nuclear antigen. Science. 1999; 284:641-4. https://doi.org/10.1126/science.284.5414.641. [PubMed]

12. Cotter MA 2nd, Robertson ES. The latency-associated nuclear antigen tethers the Kaposi's sarcoma-associated herpesvirus genome to host chromosomes in body cavitybased lymphoma cells. Virology. 1999; 264:254-64. https:// doi.org/10.1006/viro.1999.9999. [PubMed]

13. Hu J, Garber AC, Renne R. The latency-associated nuclear antigen of Kaposi's sarcoma-associated herpesvirus supports 
latent DNA replication in dividing cells. J Virol. 2002; 76:11677-87. $\quad$ https://doi.org/10.1128/jvi.76.22.1167711687.2002. [PubMed]

14. Juillard F, Tan M, Li S, Kaye KM. Kaposi's Sarcoma Herpesvirus Genome Persistence. Front Microbiol. 2016; 7:1149. https://doi.org/10.3389/fmicb.2016.01149. [PubMed]

15. Ballestas ME, Kaye KM. The latency-associated nuclear antigen, a multifunctional protein central to Kaposi's sarcoma-associated herpesvirus latency. Future Microbiol. 2011; 6:1399-413. https://doi.org/10.2217/fmb.11.137. [PubMed]

16. Weidner-Glunde M, Mariggio G, Schulz TF. Kaposi's Sarcoma-Associated Herpesvirus Latency-Associated Nuclear Antigen: Replicating and Shielding Viral DNA during Viral Persistence. J Virol. 2017; 91:e01083-16. https://doi.org/10.1128/JVI.01083-16. [PubMed]

17. De Leo A, Calderon A, Lieberman PM. Control of Viral Latency by Episome Maintenance Proteins. Trends Microbiol. 2020; 28:150-62. https://doi.org/10.1016/j. $\underline{\text { tim.2019.09.002. [PubMed] }}$

18. Lu F, Day L, Gao SJ, Lieberman PM. Acetylation of the latency-associated nuclear antigen regulates repression of Kaposi's sarcoma-associated herpesvirus lytic transcription. J Virol. 2006; 80:5273-82. https://doi.org/10.1128/ JVI.02541-05. [PubMed]

19. An FQ, Compitello N, Horwitz E, Sramkoski M, Knudsen ES, Renne R. The latency-associated nuclear antigen of Kaposi's sarcoma-associated herpesvirus modulates cellular gene expression and protects lymphoid cells from p16 INK4A-induced cell cycle arrest. J Biol Chem. 2005; 280:3862-74. https://doi.org/10.1074/jbc.M407435200. [PubMed]

20. Verma SC, Borah S, Robertson ES. Latency-associated nuclear antigen of Kaposi's sarcoma-associated herpesvirus up-regulates transcription of human telomerase reverse transcriptase promoter through interaction with transcription factor Sp1. J Virol. 2004; 78:10348-59. https://doi. org/10.1128/JVI.78.19.10348-10359.2004. [PubMed]

21. Schneider JW, Dittmer DP. Diagnosis and Treatment of Kaposi Sarcoma. Am J Clin Dermatol. 2017; 18:529-39. https://doi.org/10.1007/s40257-017-0270-4. [PubMed]

22. Garber AC, Hu J, Renne R. Latency-associated nuclear antigen (LANA) cooperatively binds to two sites within the terminal repeat, and both sites contribute to the ability of LANA to suppress transcription and to facilitate DNA replication. J Biol Chem. 2002; 277:27401-11. https://doi. org/10.1074/jbc.M203489200. [PubMed]

23. Cotter MA 2nd, Subramanian C, Robertson ES. The Kaposi's sarcoma-associated herpesvirus latency-associated nuclear antigen binds to specific sequences at the left end of the viral genome through its carboxy-terminus. Virology. 2001; 291:241-59. https://doi.org/10.1006/viro.2001.1202. [PubMed]
24. Ballestas ME, Kaye KM. Kaposi's sarcoma-associated herpesvirus latency-associated nuclear antigen 1 mediates episome persistence through cis-acting terminal repeat (TR) sequence and specifically binds TR DNA. J Virol. 2001; 75:3250-8. https://doi.org/10.1128/JVI.75.7.32503258.2001. [PubMed]

25. Domsic JF, Chen HS, Lu F, Marmorstein R, Lieberman PM. Molecular basis for oligomeric-DNA binding and episome maintenance by KSHV LANA. PLoS Pathog. 2013; 9:e1003672. https://doi.org/10.1371/journal.ppat.1003672. [PubMed]

26. Hellert J, Weidner-Glunde M, Krausze J, Richter U, Adler H, Fedorov R, Pietrek M, Ruckert J, Ritter C, Schulz TF, Luhrs T. A structural basis for BRD2/4-mediated host chromatin interaction and oligomer assembly of Kaposi sarcoma-associated herpesvirus and murine gammaherpesvirus LANA proteins. PLoS Pathog. 2013; 9:e1003640. https://doi.org/10.1371/journal.ppat.1003640. [PubMed]

27. Hellert J, Weidner-Glunde M, Krausze J, Lunsdorf H, Ritter C, Schulz TF, Luhrs T. The 3D structure of Kaposi sarcoma herpesvirus LANA C-terminal domain bound to DNA. Proc Natl Acad Sci U S A. 2015; 112:6694-9. https://doi. org/10.1073/pnas.1421804112. [PubMed]

28. Barbera AJ, Chodaparambil JV, Kelley-Clarke B, Joukov V, Walter JC, Luger K, Kaye KM. The nucleosomal surface as a docking station for Kaposi's sarcoma herpesvirus LANA. Science. 2006; 311:856-61. https://doi.org/10.1126/ science.1120541. [PubMed]

29. Beauchemin C, Moerke NJ, Faloon P, Kaye KM. Assay Development and High-Throughput Screening for Inhibitors of Kaposi's Sarcoma-Associated Herpesvirus N-Terminal Latency-Associated Nuclear Antigen Binding to Nucleosomes. J Biomol Screen. 2014; 19:947-58. https:// doi.org/10.1177/1087057114520973. [PubMed]

30. Kirsch P, Jakob V, Elgaher WAM, Walt C, Oberhausen K, Schulz TF, Empting M. Discovery of Novel LatencyAssociated Nuclear Antigen Inhibitors as Antiviral Agents Against Kaposi's Sarcoma-Associated Herpesvirus. ACS Chem Biol. 2020; 15:388-95. https://doi.org/10.1021/ acschembio.9b00845. [PubMed]

31. Kirsch P, Jakob V, Oberhausen K, Stein SC, Cucarro I, Schulz TF, Empting M. Fragment-Based Discovery of a Qualified Hit Targeting the Latency-Associated Nuclear Antigen of the Oncogenic Kaposi's SarcomaAssociated Herpesvirus/Human Herpesvirus 8. J Med Chem. 2019; 62:3924-39. https://doi.org/10.1021/acs. jmedchem.8b01827. [PubMed]

32. Messick TE, Tolvinski L, Zartler ER, Moberg A, Frostell A, Smith GR, Reitz AB, Lieberman PM. Biophysical Screens Identify Fragments That Bind to the Viral DNA-Binding Proteins EBNA1 and LANA. Molecules. 2020; 25:1760. https://doi.org/10.3390/molecules25071760. [PubMed]

33. Tso FY, West JT, Wood C. Reduction of Kaposi's SarcomaAssociated Herpesvirus Latency Using CRISPR-Cas9 To Edit 
the Latency-Associated Nuclear Antigen Gene. J Virol. 2019; 93:e02183-18. https://doi.org/10.1128/JVI.02183-18. [PubMed]

34. Ye FC, Zhou FC, Yoo SM, Xie JP, Browning PJ, Gao SJ. Disruption of Kaposi's sarcoma-associated herpesvirus latent nuclear antigen leads to abortive episome persistence. J Virol. 2004; 78:11121-9. https://doi.org/10.1128/ JVI.78.20.11121-11129.2004. [PubMed]

35. Gruffaz M, Zhou S, Vasan K, Rushing T, Michael QL, Lu C, Jung JU, Gao SJ. Repurposing Cytarabine for Treating Primary Effusion Lymphoma by Targeting Kaposi's Sarcoma-Associated Herpesvirus Latent and Lytic Replications. mBio. 2018; 9:e0756-18. https://doi. org/10.1128/mBio.00756-18. [PubMed]

36. Sin SH, Roy D, Wang L, Staudt MR, Fakhari FD, Patel DD, Henry D, Harrington WJ Jr, Damania BA, Dittmer DP. Rapamycin is efficacious against primary effusion lymphoma (PEL) cell lines in vivo by inhibiting autocrine signaling. Blood. 2007; 109:2165-73. https://doi. org/10.1182/blood-2006-06-028092. [PubMed]

37. Nagasawa J, Mizokami A, Koshida K, Yoshida S, Naito K, Namiki M. Novel HER2 selective tyrosine kinase inhibitor, TAK-165, inhibits bladder, kidney and androgenindependent prostate cancer in vitro and in vivo. Int $\mathrm{J}$ Urol. 2006; 13:587-92. https://doi.org/10.1111/j.14422042.2006.01342.x. [PubMed]

38. Grygielewicz P, Dymek B, Bujak A, Gunerka P, Stanczak A, Lamparska-Przybysz M, Wieczorek M, Dzwonek K, Zdzalik D. Epithelial-mesenchymal transition confers resistance to selective FGFR inhibitors in SNU-16 gastric cancer cells. Gastric Cancer. 2016; 19:53-62. https://doi. org/10.1007/s10120-014-0444-1. [PubMed]

39. Baccelli I, Gareau Y, Lehnertz B, Gingras S, Spinella JF, Corneau S, Mayotte N, Girard S, Frechette M, BlouinChagnon V, Leveille K, Boivin I, MacRae T, et al. Mubritinib Targets the Electron Transport Chain Complex I and Reveals the Landscape of OXPHOS Dependency in Acute Myeloid Leukemia. Cancer Cell. 2019; 36:84-99 e8. https://doi.org/10.1016/j.ccell.2019.06.003. [PubMed]

40. Narkhede M, Arora S, Ujjani C. Primary effusion lymphoma: current perspectives. Onco Targets Ther. 2018; 11:3747-54. https://doi.org/10.2147/OTT.S167392. [PubMed]

41. Fujimuro M, Liu J, Zhu J, Yokosawa H, Hayward SD. Regulation of the interaction between glycogen synthase kinase 3 and the Kaposi's sarcoma-associated herpesvirus latency-associated nuclear antigen. J Virol. 2005; 79:1042941. https://doi.org/10.1128/JVI.79.16.10429-10441.2005. [PubMed]

42. Chen W, Sin SH, Wen KW, Damania B, Dittmer DP. Hsp90 inhibitors are efficacious against Kaposi Sarcoma by enhancing the degradation of the essential viral gene LANA, of the viral co-receptor EphA2 as well as other client proteins. PLoS Pathog. 2012; 8:e1003048. https:// doi.org/10.1371/journal.ppat.1003048. [PubMed]

43. Long C, Guo W, Zhou H, Wang J, Wang H, Sun X. Triptolide decreases expression of latency-associated nuclear antigen 1 and reduces viral titers in Kaposi's sarcoma-associated and herpesvirus-related primary effusion lymphoma cells. Int J Oncol. 2016; 48:1519-30. https://doi.org/10.3892/ijo.2016.3353. [PubMed]

44. Masud Alam M, Kariya R, Kawaguchi A, Matsuda K, Kudo E, Okada S. Inhibition of autophagy by chloroquine induces apoptosis in primary effusion lymphoma in vitro and in vivo through induction of endoplasmic reticulum stress. Apoptosis. 2016; 21:1191-201. https://doi.org/10.1007/ s10495-016-1277-7. [PubMed]

45. Baba Y, Shigemi Z, Hara N, Moriguchi M, Ikeda M, Watanabe T, Fujimuro M. Arctigenin induces the apoptosis of primary effusion lymphoma cells under conditions of glucose deprivation. Int J Oncol. 2018; 52:505-17. https:// doi.org/10.3892/ijo.2017.4215. [ubMed]

46. Paul AG, Sharma-Walia N, Chandran B. Targeting KSHV/ HHV-8 latency with COX-2 selective inhibitor nimesulide: a potential chemotherapeutic modality for primary effusion lymphoma. PLoS One. 2011; 6:e24379. https://doi. org/10.1371/journal.pone.0024379. [PubMed]

47. Ding L, Zhu Q, Zhou F, Tan H, Xu W, Pan C, Zhu C, Wang Y, Zhang H, Fu W, Qian Z, Yuan Z, Xu H, et al. Identification of viral SIM-SUMO2-interaction inhibitors for treating primary effusion lymphoma. PLoS Pathog. 2019; 15:e1008174. https://doi.org/10.1371/journal.ppat.1008174. [PubMed]

48. Dai L, Bai A, Smith CD, Rodriguez PC, Yu F, Qin Z. ABC294640, A Novel Sphingosine Kinase 2 Inhibitor, Induces Oncogenic Virus-Infected Cell Autophagic Death and Represses Tumor Growth. Mol Cancer Ther. 2017; 16:2724-34. https://doi.org/10.1158/1535-7163.MCT-170485. [PubMed]

49. Qin Z, Dai L, Trillo-Tinoco J, Senkal C, Wang W, Reske T, Bonstaff K, Del Valle L, Rodriguez P, Flemington E, Voelkel-Johnson C, Smith CD, Ogretmen B, Parsons C. Targeting sphingosine kinase induces apoptosis and tumor regression for KSHV-associated primary effusion lymphoma. Mol Cancer Ther. 2014; 13:154-64. https://doi. org/10.1158/1535-7163.MCT-13-0466. [PubMed]

50. Lan K, Murakami M, Bajaj B, Kaul R, He Z, Gan R, Feldman M, Robertson ES. Inhibition of KSHV-infected primary effusion lymphomas in NOD/SCID mice by gamma-secretase inhibitor. Cancer Biol Ther. 2009; 8:213643. https://doi.org/10.4161/cbt.8.22.9743. [PubMed]

51. Saji C, Higashi C, Niinaka Y, Yamada K, Noguchi K, Fujimuro M. Proteasome inhibitors induce apoptosis and reduce viral replication in primary effusion lymphoma cells. Biochem Biophys Res Commun. 2011; 415:573-8. https:// doi.org/10.1016/j.bbrc.2011.10.107. [PubMed]

52. Mohanty S, Kumar A, Das P, Sahu SK, Choudhuri T. Multi-targeted therapy of everolimus in Kaposi's sarcoma associated herpes virus infected primary effusion lymphoma. Apoptosis. 2017; 22:1098-115. https://doi. org/10.1007/s10495-017-1391-1. [PubMed]

53. El Hajj H, Ali J, Ghantous A, Hodroj D, Daher A, Zibara K, Journo C, Otrock Z, Zaatari G, Mahieux R, El Sabban 
M, Bazarbachi A, Abou Merhi R. Combination of arsenic and interferon-alpha inhibits expression of KSHV latent transcripts and synergistically improves survival of mice with primary effusion lymphomas. PLoS One. 2013; 8:e79474. https://doi.org/10.1371/journal.pone.0079474. [PubMed]

54. Abou-Merhi R, Khoriaty R, Arnoult D, El Hajj H, Dbouk H, Munier S, El-Sabban ME, Hermine O, Gessain A, de The H, Mahieux R, Bazarbachi A. PS-341 or a combination of arsenic trioxide and interferon-alpha inhibit growth and induce caspase-dependent apoptosis in $\mathrm{KSHV} / \mathrm{HHV}$ 8 -infected primary effusion lymphoma cells. Leukemia. 2007; 21:1792-801. https://doi.org/10.1038/sj.leu.2404797. [PubMed]

55. Sarek G, Kurki S, Enback J, Iotzova G, Haas J, Laakkonen P, Laiho M, Ojala PM. Reactivation of the p53 pathway as a treatment modality for KSHV-induced lymphomas. J Clin Invest. 2007; 117:1019-28. https://doi.org/10.1172/ JCI30945. [PubMed]

56. Godfrey A, Anderson J, Papanastasiou A, Takeuchi Y, Boshoff C. Inhibiting primary effusion lymphoma by lentiviral vectors encoding short hairpin RNA. Blood. 2005; 105:2510-8. https://doi.org/10.1182/blood-2004-08-3052. [PubMed]

57. Manzano M, Patil A, Waldrop A, Dave SS, Behdad A, Gottwein E. Gene essentiality landscape and druggable oncogenic dependencies in herpesviral primary effusion lymphoma. Nat Commun. 2018; 9:3263. https://doi. org/10.1038/s41467-018-05506-9. [PubMed]

58. Lagunoff M. Activation of cellular metabolism during latent Kaposi's Sarcoma herpesvirus infection. Curr Opin Virol. 2016; 19:45-9. https://doi.org/10.1016/j. coviro.2016.06.012. [PubMed]

59. Delgado T, Carroll PA, Punjabi AS, Margineantu D, Hockenbery DM, Lagunoff M. Induction of the Warburg effect by Kaposi's sarcoma herpesvirus is required for the maintenance of latently infected endothelial cells. Proc Natl Acad Sci U S A. 2010; 107:10696-701. https://doi. org/10.1073/pnas.1004882107. [PubMed]
60. Zhu Y, Ramos da Silva S, He M, Liang Q, Lu C, Feng P, Jung JU, Gao SJ. An Oncogenic Virus Promotes Cell Survival and Cellular Transformation by Suppressing Glycolysis. PLoS Pathog. 2016; 12:e1005648. https://doi. org/10.1371/journal.ppat.1005648. [PubMed]

61. Gjyshi O, Bottero V, Veettil MV, Dutta S, Singh VV, Chikoti L, Chandran B. Kaposi's sarcoma-associated herpesvirus induces Nrf2 during de novo infection of endothelial cells to create a microenvironment conducive to infection. PLoS Pathog. 2014; 10:e1004460. https://doi.org/10.1371/journal. ppat.1004460. [PubMed]

62. Ma Q, Cavallin LE, Leung HJ, Chiozzini C, GoldschmidtClermont PJ, Mesri EA. A role for virally induced reactive oxygen species in Kaposi's sarcoma herpesvirus tumorigenesis. Antioxid Redox Signal. 2013; 18:80-90. https://doi.org/10.1089/ars.2012.4584. [PubMed]

63. Caro-Vegas C, Bailey A, Bigi R, Damania B, Dittmer DP. Targeting mTOR with MLN0128 Overcomes Rapamycin and Chemoresistant Primary Effusion Lymphoma. mBio. 2019; 10:e02871-18. https://doi.org/10.1128/mBio.0287118. [PubMed]

64. Sin SH, Eason AB, Bigi R, Kim Y, Kang S, Tan K, Seltzer TA, Venkataramanan R, An H, Dittmer DP. Kaposi's Sarcoma-Associated Herpesvirus Latency Locus Renders B Cells Hyperresponsive to Secondary Infections. J Virol. 2018; 92:e01138-18. https://doi.org/10.1128/JVI.01138-18. [PubMed]

65. Khandelia P, Yap K, Makeyev EV. Streamlined platform for short hairpin RNA interference and transgenesis in cultured mammalian cells. Proc Natl Acad Sci U S A. 2011; 108:12799-804. https://doi.org/10.1073/pnas.1103532108. [PubMed] 